\title{
Ceres observed at low phase angles by VIR-Dawn
}

\author{
M. Ciarniello ${ }^{1}$, M. C. De Sanctis ${ }^{1}$, A. Raponi ${ }^{1}$, B. Rousseau ${ }^{1}$, A. Longobardo ${ }^{1}$, J.-Y. Li $^{2}$, S. E. Schröder ${ }^{3}$, F. Tosi ${ }^{1}$, \\ F. Zambon ${ }^{1}$, E. Ammannito ${ }^{4}$, F. G. Carrozzo ${ }^{1}$, A. Frigeri ${ }^{1}$, E. Rognini ${ }^{5}$, C. A. Raymond ${ }^{6}$, and C. T. Russell ${ }^{7}$ \\ ${ }^{1}$ IAPS-INAF, Via Fosso del Cavaliere, 100, 00133 Rome, Italy \\ e-mail: mauro.ciarniello@inaf.it \\ 2 Planetary Science Institute, Tucson, AZ, USA \\ ${ }^{3}$ German Aerospace Center DLR, Institute of Planetary Research, Berlin, Germany \\ ${ }^{4}$ ASI, Rome, Italy \\ 5 ASI-SSDC, Rome, Italy \\ ${ }^{6}$ Jet Propulsion Laboratory, California Institute of Technology, Pasadena, USA \\ ${ }^{7}$ University of California Los Angeles, Earth Planetary and Space Sciences, Los Angeles, CA, USA
}

Received 9 August 2019 / Accepted 11 December 2019

\begin{abstract}
Context. Particulate surfaces exhibit a surge of reflectance at low phase angles, a phenomenon referred to as the opposition effect (OE). Two mechanisms are recognized as responsible for the OE: shadow hiding $(\mathrm{SH})$ and coherent backscattering. The latter is typically characterized by a small angular width of a few degrees at most and according to the theoretical prediction should exhibit wavelength and albedo dependence.

Aims. We characterize the OE on the surface of Ceres using Dawn Visible InfraRed mapping spectrometer hyperspectral images at low phase angles. Furthermore, this dataset, coupled with previous observations, allows us to perform a complete spectrophotometric modeling at visual-to-infrared (VIS-IR) wavelengths $(0.465-4.05 \mu \mathrm{m})$ in the broad phase angle range $\approx 0^{\circ}-132^{\circ}$.

Methods. We applied Hapke's theory to the average phase curve for Ceres. Disk-resolved properties of the OE were investigated through an empirical model.

Results. Across the investigated phase angle interval, Ceres' average phase curve exhibits a smaller back-scattering contribution for increasing wavelengths. This determines a progressive spectral reddening at larger phase angles that we hypothesize as being related to the effect of submicron roughness on the grain surface. In the OE region, the shape of the phase curves is fairly constant across the VIS range and no sharp opposition surge at very small phase angles $\left(\alpha<2^{\circ}\right)$ can be recognized. This would suggest a major contribution from SH to Ceres' OE. Assuming SH as the dominant mechanism, from the OE angular width we infer a high surface porosity ( $\approx 0.9)$, which appears in good qualitative agreement with Ceres' low thermal inertia. Thanks to the OE observations we derive Ceres' VIS-IR geometric albedo with a reference value at $0.55 \mu \mathrm{m}$ of $0.098 \pm 0.007$. Mapping of the VIS normal albedo and OE angular width across a portion of the surface of Ceres does not reveal a spatial correlation between these quantities, consistent with SH dominating in the $\alpha=0^{\circ}-7^{\circ}$ interval. The comparison of Ceres' $V$-band magnitude curve with that of other asteroids indicates that Ceres' OE is typical of a low-albedo object and compatible with the C-class type.
\end{abstract}

Key words. minor planets, asteroids: individual: Ceres - methods: data analysis - techniques: photometric - radiative transfer techniques: imaging spectroscopy - planets and satellites: surfaces

\section{Introduction}

The NASA-Dawn spacecraft extensively observed the surface of Ceres from early 2015, approximately $3 \mathrm{yr}$ after its departure from Vesta, to the end of the mission on 1 November 2018. It was equipped with two imaging instruments: the Framing Camera (FC; Sierks et al. 2011) and the Visible InfraRed mapping spectrometer (VIR; De Sanctis et al. 2011). The large dataset produced by the Dawn mission revealed a dark object (geometric albedo of about 0.09-0.1 at visible wavelengths; Ciarniello et al. 2017; Schröder et al. 2017; Li et al. 2019) characterized by an almost neutral spectral slope at visible wavelengths longward of $0.5 \mu \mathrm{m}$. Conversely, several absorption features show up in the infrared part of the spectrum, indicating the presence of widespread $\mathrm{Mg}$-bearing and ammoniated phyllosilicates, and $\mathrm{Ca}-\mathrm{Mg}$ carbonates across the surface, mixed with a lowalbedo endmember (De Sanctis et al. 2015; Ammannito et al. 2016). The overall dark surface of Ceres hosts isolated "bright spots" (with visual normal albedo up to six times Ceres' average;
Schröder et al. 2017), the most notable ones being located in the Occator crater (Cerealia and Vinalia faculae), showing the occurrence of large amounts of Na-Carbonates (De Sanctis et al. 2016; Palomba et al. 2019; Raponi et al. 2019a). Additional smallscale compositional variability was evidenced by the presence of localized exposed water ice (Combe et al. 2016; Raponi et al. 2018) and organics (De Sanctis et al. 2017, 2018; Kaplan et al. 2018; Raponi et al. 2019b).

The Dawn observation campaign was composed of a series of sequences, mostly characterized by different altitudes of the spacecraft over the surface, which allowed Ceres to be investigated with different spatial resolutions and a large variety of observation geometries. In particular, a thorough characterization of the dwarf planet spectrophotometric properties was performed by means of FC observations acquired during the Rotational Characterization 3 (RC3, spatial resolution of $\left.\approx 1.3 \mathrm{~km} \mathrm{pixel}^{-1}\right)$, Survey $\left(0.45 \mathrm{~km} \mathrm{pixel}^{-1}\right)$ and High Altitude Mapping Orbit (HAMO, $0.14 \mathrm{~km}$ pixel $^{-1}$ ) by Schröder et al. (2017) and Li et al. (2019), while VIR hyperspectral images 
acquired from the Approach down to the Survey Phase (spatial resolution varying from $\approx 90$ to $\approx 1 \mathrm{~km} \mathrm{pixel}^{-1}$ ) have been used in Ciarniello et al. (2017), and, with the inclusion of HAMO $\left(\approx 400 \mathrm{~m} \mathrm{pixel}^{-1}\right)$ and Low Mapping Altitude Orbit (LAMO, $\approx 100 \mathrm{~m} \mathrm{pixel}^{-1}$ ) data, by Longobardo et al. (2019). Overall, these studies investigated Ceres' reflectance properties in a fairly large phase angle interval, extending from approximately $7^{\circ}$ to $132^{\circ}$.

Following the end of the nominal mission (18 June 2016), Dawn operations went through a series of extensions, and on 29 April 2017, during the Extended Mission Orbit 4 (XMO4), a dedicated maneuver allowed the imaging instruments to observe Ceres at opposition, thus exploring the low-phase-angle part of the phase curve, which until that time had only been investigated by ground-based observations (Tedesco et al. 1989; Reddy et al. 2015), and where a phenomenon known as the opposition effect $(\mathrm{OE})$ takes place. The $\mathrm{OE}$ is a surge in reflectance commonly observed in particulate media at small phase angles (Hapke 2012), and has been widely observed on atmosphereless bodies of the Solar System, such as the Moon (Buratti et al. 1996; Helfenstein et al. 1997; Shkuratov et al. 1999), asteroids (Belskaya \& Shevchenko 2000; Domingue et al. 2002; Kitazato et al. 2008; Spjuth et al. 2012; Masoumzadeh et al. 2015; Shevchenko et al. 2016), comets (Ciarniello et al. 2015; Hasselmann et al. 2017), icy moons (Helfenstein et al. 1998; Pitman et al. 2010; Ciarniello et al. 2011), and rings (Déau et al. 2013; Salo \& French 2010), as well as in laboratory measurements (Nelson et al. 2000; Shepard \& Helfenstein 2011; Jost et al. 2017). Two mechanisms are proposed as the main contributors to this effect: the shadow hiding OE (SHOE) and the coherent backscattering OE (CBOE). In a particulate medium such as the regolith on the surface of a planetary object, SHOE is produced by particles on the top layers that progressively hide their own shadow at decreasing phase angles, thus limiting the visible shadows cast on the particles below (Hapke 2012). Alternatively, $\mathrm{CBOE}$ is explained by the constructive interference of light wavefronts (maximal at $0^{\circ}$ phase angle) propagating within the medium along the same paths but in opposite directions (Shkuratov et al. 1999; Mishchenko et al. 2009). Generally, CBOE is considered to be characterized by a narrow (few degree wide or less) peak (Mishchenko 1992; Nelson et al. 2002), while that of SHOE is typically broader (Hapke 2012).

Recently, FC images at opposition were investigated in Schröder et al. (2018) to characterize Ceres' OE. In this work, we take advantage of the observations acquired by the instrument VIR on 29 April 2017 (Sect. 2) to integrate the previous dataset used in Ciarniello et al. (2017) and provide an updated spectrophotometric model of the surface of Ceres at VIS-IR wavelengths in the broad $\approx 0^{\circ}-132^{\circ}$ phase angle interval by means of the Hapke model (Hapke 2012; Sect. 3). In addition, we investigate OE spatial variability across the surface (Sect. 4) and compare the phase curve for Ceres with OE measurements performed on different classes of asteroids (Sect. 5). Finally, a summary of the main findings of this work is given in Sect. 6.

\section{Dataset}

The VIR instrument (De Sanctis et al. 2011) is composed of two separate channels observing the same target in two different wavelength ranges: the visible channel (VIS) and the infrared channel (IR), covering the intervals $0.25-1.05 \mu \mathrm{m}$ and $1-5.1 \mu \mathrm{m}$, respectively. For both channels, the image of the target is acquired through the spectrometer slit across 256 pixels (samples) with an instantaneous field of view (IFOV) of $250 \mu \mathrm{rad} \times 250 \mu \mathrm{rad}$, forming an imaging line. During the line acquisition, the spectrum of each sample is measured. Different lines are then combined together to form a hyperspectral image or cube, containing images of the target at different wavelengths (bands). The VIS and IR channels both have 432 bands, with a nominal spectral sampling of 1.8 and $9.5 \mathrm{~nm}$, respectively.

On 29 April 2017, VIR acquired a series of 19 VIS images and a single IR image of Ceres during the OE maneuver from a distance of $\approx 20000 \mathrm{~km}$, which corresponds to a spatial resolution at the surface of $\approx 5 \mathrm{~km} \mathrm{pix}^{-1}$. The projection of the FOVs of the acquisitions is shown in Fig. 1. In the case of the VIS channel, the phase angle of the observations spans from $\approx 0^{\circ}$ to $\approx 6^{\circ}$, while, given the $3.6^{\circ}$-wide FOV of the instrument, the single IR cube covers the $\approx 0^{\circ}-1.6^{\circ}$ interval. The list of VIR acquisitions, which we generally refer to as "OE observations" hereafter, is given in Table 1. This set of hyperspectral cubes, when integrated with the data acquired during previous phases of the Dawn mission at Ceres with comparable spatial resolution (Approach Phase, RC3, Transfer to Survey, Survey) already analyzed in Ciarniello et al. (2017), allow us to provide a thorough characterization of the global reflectance curve for Ceres, filling the observational gap at low phase angles of our previous study for the VIS wavelengths and partly for the IR. This is shown in Fig. 2, where we report the radiance factor $\left(I / F^{1}\right)$ at $0.55 \mu \mathrm{m}$ as measured by VIR against phase angle $(\alpha)$, combining the OE observations with the dataset discussed by Ciarniello et al. (2017), encompassing approximately the $0^{\circ}-132^{\circ}$ interval. According to the spectral capabilities of VIR, similar reflectance curves are derived across the entire $0.465-4.05 \mu \mathrm{m}$ spectral range, corresponding to the interval with the best signal-to-noise ratio (S/N), after correction for spectral artifacts (Raponi 2015; Carrozzo et al. 2016) and IR thermal emission (Raponi et al. 2019b).

\section{Hapke modeling and the phase curve of Ceres}

With the aim of constraining the physical properties of the regolith covering the surface of Ceres, we apply Hapke's model (Hapke 2012) to VIR observations following the approach described in Ciarniello et al. (2017). In this case, the radiance factor of the observed surface is expressed by:

$$
\begin{aligned}
\frac{I}{F}= & \frac{w}{4} \frac{\mu_{0 e}}{\mu_{0 e}+\mu_{e}} \\
& \times\left[\left(1+B_{\mathrm{SH}}(\alpha)\right) p(\alpha)+H\left(w, \mu_{0 e}\right) H\left(w, \mu_{e}\right)-1\right] \\
& \times\left[1+B_{\mathrm{CB}}(\alpha)\right] S(i, e, \alpha, \bar{\theta}),
\end{aligned}
$$

with

$-w$ : single scattering albedo (SSA);

- $p(\alpha)$ : single particle phase function (SPPF);

- $H\left(w, \mu_{0 e}\right), H\left(w, \mu_{e}\right)$ : Chandrasekhar functions computed at $\mu_{0 e}$ and $\mu_{0}$;

- $S(i, e, \alpha, \bar{\theta})$ : surface roughness term depending on the average surface slope parameter $\bar{\theta}$;

- $B_{\mathrm{SH}}(\alpha)$ : SHOE term, depending on SHOE amplitude $\left(B_{0 \mathrm{SH}}\right)$ and angular width $\left(h_{\mathrm{SH}}\right)$;

- $B_{\mathrm{CB}}(\alpha)$ : CBOE term, depending on CBOE amplitude $\left(B_{0 \mathrm{CB}}\right)$ and angular width $\left(h_{\mathrm{CB}}\right)$;

1 Radiance factor is defined as the ratio between the radiance $(I)$ scattered by the target surface and the radiance of a perfect Lambertian surface perpendicularly illuminated $(F)$. Defining the Solar irradiance at the target as $J$, the surface bidirectional reflectance as $r$, and being the Lambertian reflectance at null incidence angle $1 / \pi$, it gives $I=J r$, $F=J / \pi$ and $I / F=\pi r$. 

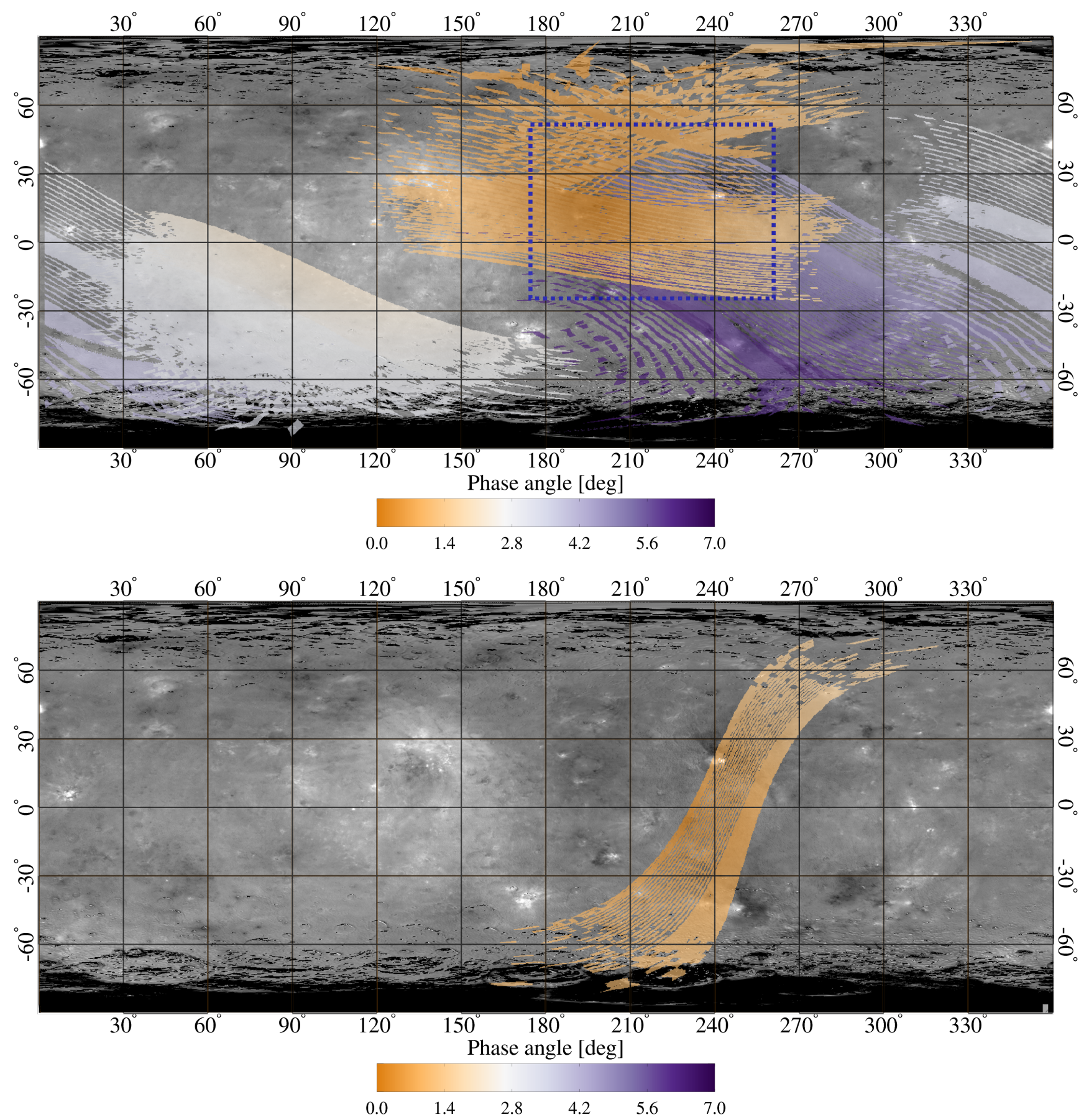

Fig. 1. Top panel: FOVs of the 19 VIS hyperspectral cubes acquired during the OE maneuver projected on Ceres FC clear filter albedo map (Schröder et al. 2017). The color bar indicates the phase angle in each pixel. Due to spacecraft motion, the different lines in a single acquisition may not be connected. The blue dashed line indicates a region investigated for disk-resolved analysis (see Sect. 4). Bottom panel: same as top panel but for the single IR hyperspectral cube.

$-\mu_{0 e}, \mu_{e}:$ cosines of the effective incidence and emission angles, respectively.

For the full analytic expression of the terms reported above and further details, we refer the reader to Hapke (2012). In analogy with the Shkuratov et al. (2011) model, we can rearrange Eq. (1) by defining a disk-function $D=\frac{2 \mu_{0 e}}{\mu_{0 e}+\mu_{e}} S(i, e, \alpha, \bar{\theta})$, and the phase curve $\frac{I / F}{D}=\frac{w}{8}\left[\left(1+B_{\mathrm{SH}}(\alpha)\right) p(\alpha)+H\left(w, \mu_{0 e}\right)\right.$ $\left.H\left(w, \mu_{e}\right)-1\right]\left[1+B_{\mathrm{CB}}(\alpha)\right]$. In the case of Ceres' surface, which is characterized by a low surface albedo, the phase curve depends mostly on the phase angle, given the minor contribution of the multiple scattering term $H\left(w, \mu_{0 e}\right) H\left(w, \mu_{e}\right)-1$ in Eq. (1), and it is representative of the intrinsic spectrophotometric properties of the regolith. Conversely, the disk-function accounts for most of the brightness variability induced by the variation of the incidence and emission angles across the surface and has neither albedo nor spectral dependence.

\subsection{Phase curve for Ceres}

The phase curve for Ceres is reported in Fig. 3 as obtained by dividing the $I / F$ of each VIR pixel by the corresponding value 
Table 1. Geometric circumstances of the OE observations performed by VIR.

\begin{tabular}{ccccc}
\hline \hline Cube SCET number & $\alpha$ coverage $[\mathrm{deg}]$ & $\langle i\rangle[\mathrm{deg}]$ & $\langle e\rangle[\mathrm{deg}]$ & $\mathrm{SR}\left[\mathrm{km} \mathrm{pix}^{-1}\right]$ \\
\hline V546731788 & $0.630-1.310$ & 60.7 & 61.6 & 5.0 \\
V546732290 & $0.644-1.344$ & 56.5 & 57.3 & 5.0 \\
V546733092 & $0.009-1.506$ & 31.0 & 31.6 & 5.0 \\
V546733894 & $0.006-1.560$ & 34.9 & 35.4 & 5.0 \\
V546734698 & $0.244-1.655$ & 32.1 & 32.7 & 5.0 \\
V546735500 & $0.010-1.639$ & 43.0 & 43.6 & 5.0 \\
V546736302 & $0.404-1.011$ & 64.9 & 65.5 & 5.0 \\
V546742558 & $2.101-2.732$ & 40.7 & 42.7 & 4.9 \\
V546743360 & $2.450-2.967$ & 47.8 & 50.3 & 4.9 \\
V546744162 & $2.815-3.201$ & 56.6 & 59.5 & 4.9 \\
V546749758 & $3.234-3.680$ & 33.8 & 36.1 & 4.9 \\
V546750560 & $3.832-4.124$ & 47.8 & 51.3 & 4.9 \\
V546751362 & $2.697-4.479$ & 40.4 & 42.1 & 4.8 \\
V546759118 & $4.753-5.215$ & 29.7 & 31.9 & 4.8 \\
V546759920 & $4.944-5.904$ & 51.7 & 56.9 & 4.8 \\
V546760722 & $4.396-5.668$ & 36.4 & 35.9 & 4.8 \\
V546762658 & $4.862-6.466$ & 39.8 & 42.8 & 4.8 \\
V546763460 & $5.158-5.651$ & 30.6 & 30.2 & 4.7 \\
V546764262 & $6.072-6.490$ & 39.3 & 44.5 & 4.8 \\
\hline I546730582 & $0.091-1.624$ & 32.5 & 33.1 & 5.0 \\
\hline
\end{tabular}

Notes. For each cube, the SCET (spacecraft elapsed time) number (V and I prefix indicate visible and infrared observations, respectively), the phase angle $\alpha$ coverage, the average incidence $i$ and emission $e$ angles, and the spatial resolution (SR) are reported.

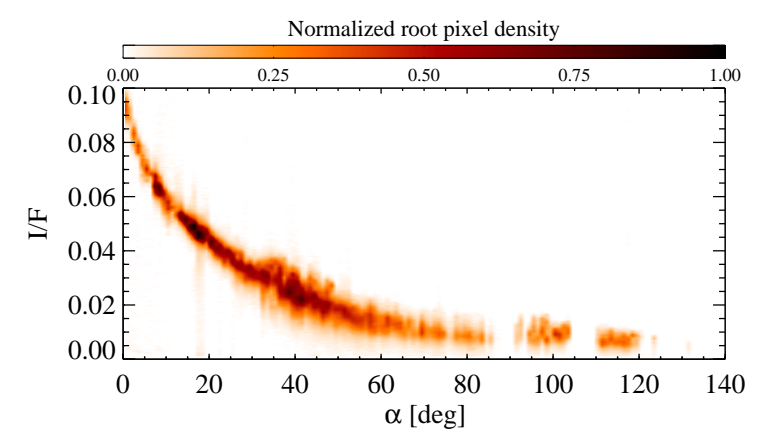

Fig. 2. $I / F$ at $0.55 \mu \mathrm{m}$ as a function of the phase angle: the contour plot shows the square root of the point density normalized to its maximum value. Data are from Approach Phase, RC3, Transfer to Survey, Survey, and $\mathrm{OE}$ observations.

of $D$ computed from the observation geometry calculated with the NAIF-SPICE toolkit (Acton 1996). Observations are grouped and averaged in phase angle bins of $1^{\circ}$ in width for $\alpha>7^{\circ}$ and phase angle bins of $0.2^{\circ}$ in width for $\alpha<7^{\circ}$ to better characterize the OE region. It can be noted that $D$, as defined here, is a function of the average surface slope $\bar{\theta}$. We assume $\bar{\theta}=29^{\circ}$, from Ciarniello et al. (2017). This choice does not affect the discussion of the spectral variability of the phase curve shape given below, although an independent determination of $\bar{\theta}$ is presented further below.

While no obvious trend with the overall albedo can be noted (Figs. 3a and c), the phase curve for Ceres gets progressively less back-scattering at larger wavelengths (Figs. $3 b$ and d), for which a reduction of the normalized $\frac{I / F}{D}$ at smaller phase angles can be observed. Such behavior produces the "phase reddening" (e.g., the increase of spectral slope with phase angle) which for Ceres was already pointed out in Ciarniello et al. (2017), Li et al. (2019) and Longobardo et al. (2019) across the VIS-IR spectral range.
Analysis. The lack of correlation between overall albedo and phase curve shape suggests that phase reddening on Ceres is mostly related to a single-grain scattering mechanism rather than on multiple scattering. This consideration is further supported by the low albedo of Ceres' surface, for which high scattering orders should provide a minor contribution to the final reflectance. As also discussed in $\mathrm{Li}$ et al. (2019) for FC observations of Ceres at VIS wavelengths, laboratory measurements (Schröder et al. 2014; Pilorget et al. 2016) and numerical simulations (Schröder et al. 2014) of the reflectance output of powders point out that the variation of the photometric response with wavelength of a given regolith can be connected, along with composition and grain size, to small-scale roughness. Schröder et al. (2014) suggest that micro-roughness, in particular at the scale of the particle surface, can explain the monotonic phase reddening observed on coarse opaque powders (basalt, in their case). Following this argument, we can argue that the same general mechanism is the cause of the phase reddening on Ceres' surface. This interpretation appears plausible when the ratio between Ceres' phase curve at $\alpha=20^{\circ}$ and $\alpha=80^{\circ}$ against wavelength is taken into consideration (Fig. 4). It can be noted that the ratio decreases from VIS to IR, in agreement with the reduced back-scattering behavior of the phase curve at larger wavelengths. Moreover, the reduction rate of the ratio progressively gets smaller, going from maximum values in the VIS to nearly zero in the IR, with only some localized variability associated with phyllosilicates and carbonate absorptions and a possible residual thermal contribution. This could indicate that Ceres' phase curve shape is decreasingly sensitive to wavelength towards the IR. If we consider the Rayleigh criterion for optical flatness ${ }^{2}\left(H<\frac{\lambda}{8 \cos \left(i_{\mathrm{p}}\right)}\right.$, where $H$ is the roughness height, $\lambda$ the wavelength, and $i_{\mathrm{p}}$ the incidence

\footnotetext{
2 Rayleigh criterion is adopted to establish whether a surface can be considered smooth to the incoming light. It defines an upper limit for the height of the surface small-scale roughness.
} 


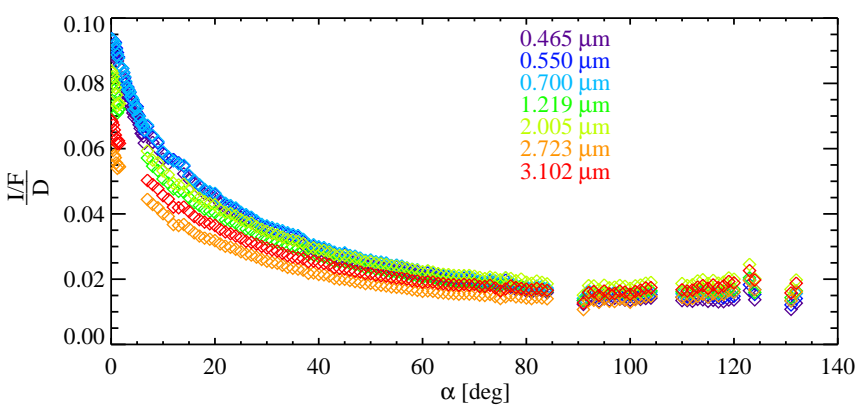

(a)

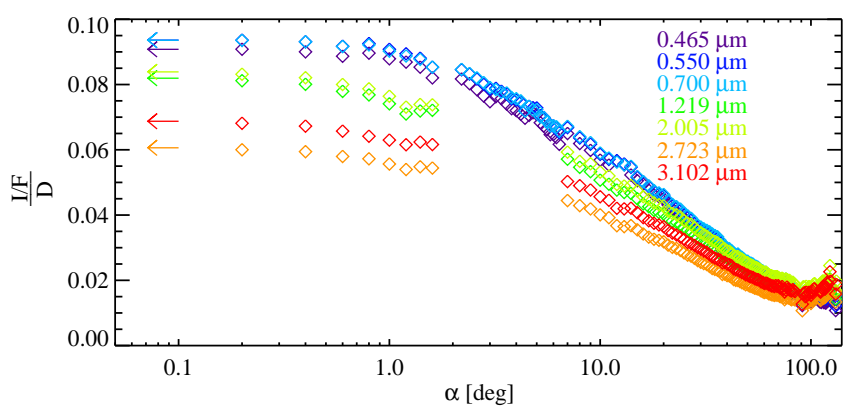

(c)

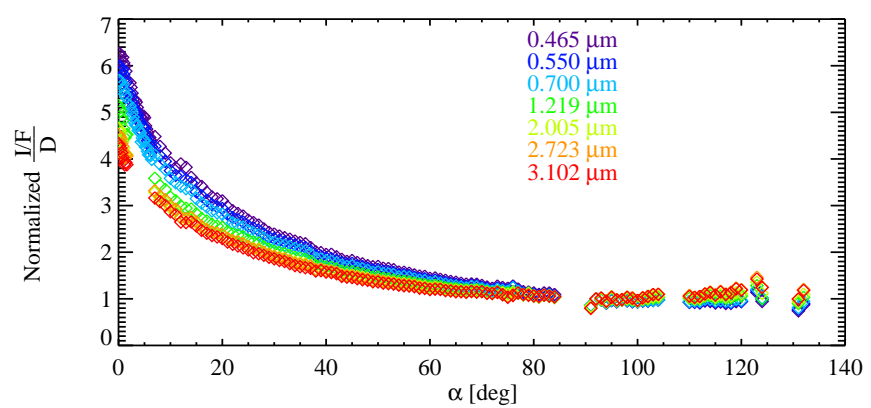

(b)

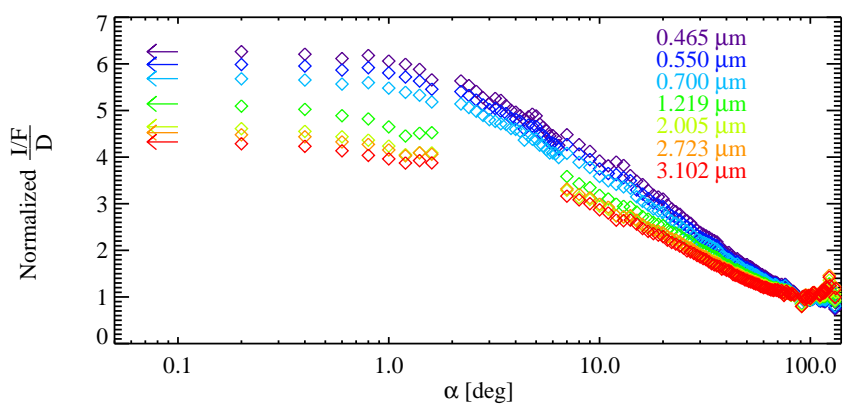

(d)

Fig. 3. Phase curves for Ceres at different wavelengths (indicated in the plot): $(a)$ absolute value; $(b)$ after normalization; $(c)$ and $(d)$ same as $(a)$ and (b) but with phase angle on logarithmic scale, respectively. In order to exploit the relative contribution in the back-scattering and forward-scattering lobes, the ideal phase angle with respect to which we normalize the phase curve would be $\alpha=90^{\circ}$. Unfortunately, this phase angle is not covered in the investigated dataset. Similarly, the closest position for which observations are provided, $\alpha=91^{\circ}$, is affected by poor statistics. Given this, the normalization in $(b)$ and $(d)$ was performed at $\alpha=92^{\circ}$. Arrows in $(c)$ and $(d)$ represent the corresponding value for the $0^{\circ}$ phase angle bin. Data are from Approach Phase, RC3, Transfer to Survey, Survey, and OE observations.

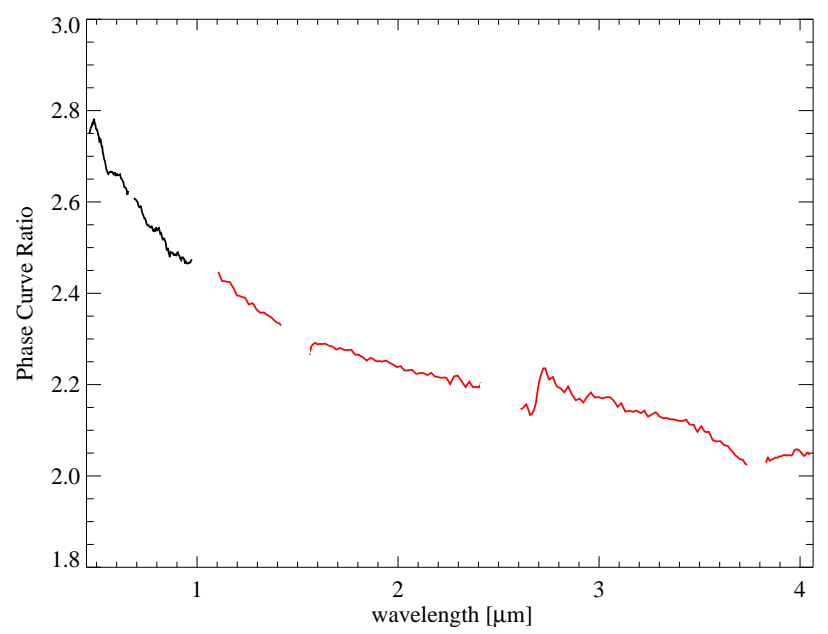

Fig. 4. Ratio between Ceres' phase curve at $\alpha=20^{\circ}$ and $\alpha=80^{\circ}$ (Phase Curve Ratio) against wavelength (VIS: black, IR: red). Gaps in the spectrum correspond to the order-sorting filter positions and VISIR junction of the instrument. We limit our analysis to $\lambda>0.465 \mu \mathrm{m}$ because of increasing instrumental noise at the shortest wavelengths and $\lambda<4.065 \mu \mathrm{m}$ because of the contribution of the thermal emission longward of this value.

angle with respect to the particle surface), such behavior would be in agreement with the assumption that small-scale roughness is related to phase reddening, and that its effect becomes progressively less important at larger wavelengths with particles appearing smoother to the incoming light. From the trend in Fig. 4, it is then possible to provide a gross estimate of the typical roughness characterizing Ceres' particles, which appears to be at submicron scale ${ }^{3}$. This is compatible with macroscopic grains on Ceres' surface, which are typically $100 \mu \mathrm{m}$ in size as derived in Raponi et al. (2019b), and can host such roughness on their surfaces. Nonetheless, we also note from Schröder et al. (2014) that fine powders of submicron grains can produce monotonic phase reddening as well, as suggested for Ceres by Li et al. (2019). This is found for laboratory samples characterized by large microscopic roughness at particle size scale (in Schröder et al. 2014, this is obtained by sprinkling the powder in the sample holder with the production of complex microscopic structures referred to as "fairy-castles", Hapke \& Van Horn 1963, due to the particular arrangements of the single grains which are controlled by Van der Waals forces). In that case, the roughness scale we derived above for Ceres would represent an estimate of the actual grain size of the regolith, which incidently may not be compatible with the grain sizes derived by spectral modeling (Raponi et al. 2019b).

\subsection{Phase curve for Ceres at low phase angles}

Figure 5 shows a close-up of Ceres's phase curve at small phase angles $\left(\alpha<10^{\circ}\right)$ after normalization at $\alpha=7^{\circ}$. In this phase angle range, the phase curves at VIS wavelengths, for which it is possible to take advantage of the complete phase angle coverage

3 From the Rayleigh criterion for optical flatness, assuming $\lambda=3 \mu \mathrm{m}$ and $\cos \left(i_{\mathrm{p}}\right)=1$, it gives $H<0.4 \mu \mathrm{m}$. 

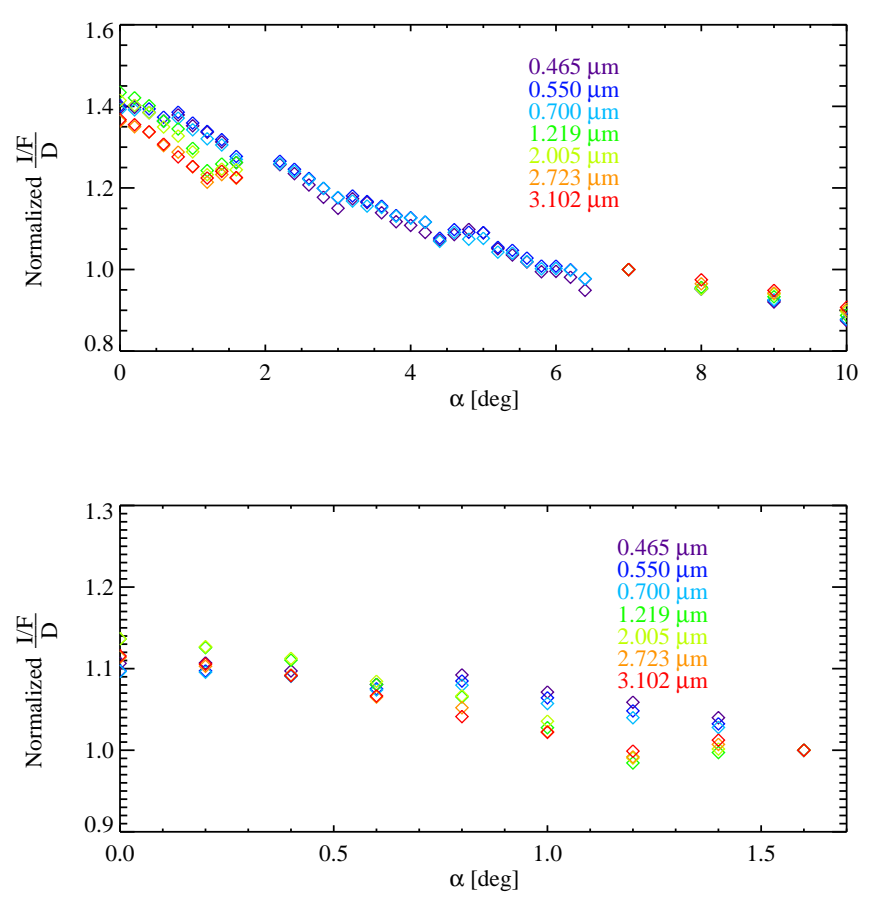

Fig. 5. Top panel: phase curves for Ceres in the $0^{\circ}-10^{\circ}$ phase angle interval at different wavelengths (indicated in the plot) after normalization at $7^{\circ}$ phase angle. Bottom panel: phase curves for Ceres in the $0^{\circ}-1.7^{\circ}$ phase angle interval at different wavelengths after normalization at $1.6^{\circ}$ phase angle.

down to $0^{\circ}$, basically overlap, showing minor spectral dependence. In the IR, moving from shorter to longer wavelengths, the phase curve exhibits some possibly systematic variability at very small phase angles $\left(<2^{\circ}\right)$ and exhibits a different slope with respect to the VIS wavelengths. The $\alpha=0^{\circ}-1.7^{\circ}$ interval of the phase curve is then further explored in Fig. 5 after normalization at $1.6^{\circ}$. This region is particularly interesting since it should be diagnostic for the detection of CBOE, which is typically characterized by a small angular width (Nelson et al. 2000). However, in terms of the shape of the OE surge, no sharp rise of the phase curve is observed, with a mostly linear increase in the photometric output towards small phase angles both in the VIS and IR cases $^{4}$.

Analysis. As mentioned above, CBOE is produced by the constructive interference of light propagating within the medium along conjugate paths (Shkuratov \& Helfenstein 2001). In particular, it can be shown that for small phase angles the phase shift of two wavelets going along the direct and the time-reversal trajectories connecting two positions on the surface is directly proportional to the phase angle and inversely proportional to the wavelength $\lambda$ (Hapke 2012). According to the theoretical treatment of Mishchenko (1992), the angular width of CBOE is characterized by a direct dependence on both wavelength and regolith albedo ${ }^{5}$. In addition, as CBOE is produced by light scattered multiple

\footnotetext{
4 We note here that the angular size of the Sun as seen by the surface of the target introduces a rounding of the OE peak. In the case of Ceres, the Sun is $\approx 0.2^{\circ}$ in width. Schröder et al. (2018) computed that this affects the phase curve for $\alpha<0.05^{\circ}$ with a maximum reduction of the phase curve of $\approx 0.5 \%$ at opposition. Given the smallness of the effect, we treated it as negligible for our purpose.

5 We also note that albedo itself is a function of wavelength, which further complicates the wavelength-dependent effects on the $\mathrm{CBOE}$ width.
}

times within the medium, a dependence of its amplitude on the albedo is in principle also expected. In this respect, the heuristic model of Shkuratov \& Helfenstein (2001) suggests that CBOE amplitude is controlled by the complex convolution of the albedo of the regolith scattering elements and their packing state, with possibly several different size scales contributing to the final $\mathrm{CBOE}$ of the particle aggregates composing planetary surfaces.

From VIR OE observations, it emerges that, for the VIS wavelength, variability in this phase angle range is basically absent, but we may wonder whether the relatively larger differences that can be noted at IR wavelengths can be linked to possible CBOE. In this respect, the changes in phase curve shape apparently correlate with wavelength, with a progressive reduction of the phase curve peak at opposition moving from 1.2 to $3.1 \mu \mathrm{m}$. Nonetheless, the fact that phase curves at $1.2-2$ and $2.7-3.1 \mu \mathrm{m}$ group separately may also suggest a dependence on intrinsic albedo, given that at $2.7-3.1 \mu \mathrm{m}$ Ceres' spectrum is characterized by phyllosilicate absorptions. The possible correlation with wavelength or with the albedo tempt an interpretation of such variability as the product of CBOE. However, with our dataset, it is not possible to disentangle this from the effect of local variability at the surface as, because of the availability of only one observation at IR wavelengths, different $\alpha$ in the phase curve sample different regions on Ceres (Fig. 1). Given this, it is highly probable that the observed changes in phase curve slope are simply the spurious effect of compositional differences between separate regions observed at different phase angles. On the contrary, the lack of this variability at VIS wavelengths, for which the coverage is more ample, and the morphology of the phase curve, missing a relatively sharp reflectance increase at small phase angles, point to a minor contribution of CBOE, suggesting $\mathrm{SH}$ as the main mechanism in the development of the OE surge.

\subsection{Hapke's model parameters}

Following Ciarniello et al. (2017), we apply Hapke's formalism to the VIR dataset to provide an updated spectrophotometric model of Ceres, by taking advantage of the OE observations, which were not available at the time of our earlier work. In particular, in the present study, we are able to constrain the OE parameters of the Hapke model, while previously they were adopted from Helfenstein \& Veverka (1989). Following the considerations of Sect. 3.2, which points to a limited contribution of $\mathrm{CB}$, we assume that $\mathrm{SH}$ is responsible for $\mathrm{OE}$ in Ceres, and the term for $\mathrm{CB}$ in Eq. (1) is set to zero $\left(B_{\mathrm{CB}}(\alpha)=0\right)$. For what concerns the SPPF, a double-lobed Henyey-Greenstein formulation is used (Hapke 2012):

$$
\begin{aligned}
p(\alpha)= & \frac{1+c}{2} \frac{1-b^{2}}{\left(1-2 b \cos (\alpha)+b^{2}\right)^{3 / 2}} \\
& +\frac{1-c}{2} \frac{1-b^{2}}{\left(1+2 b \cos (\alpha)+b^{2}\right)^{3 / 2}} .
\end{aligned}
$$

The approach adopted to fit Ceres' phase curve and determine the free parameters of the model $\left(w, B_{0 \mathrm{SH}}, h_{\mathrm{SH}}, b, c, \bar{\theta}\right)$ is described in Ciarniello et al. (2017) and is not repeated here.

Below, the new set of derived parameters is compared with the one obtained in Ciarniello et al. (2017). We refer to the latter as solution S1, while the former is defined as S2. Moreover, as described below, two further implementations of the Hapke model are investigated, leading to the additional solutions S3 and $\mathrm{S} 4$, respectively. 


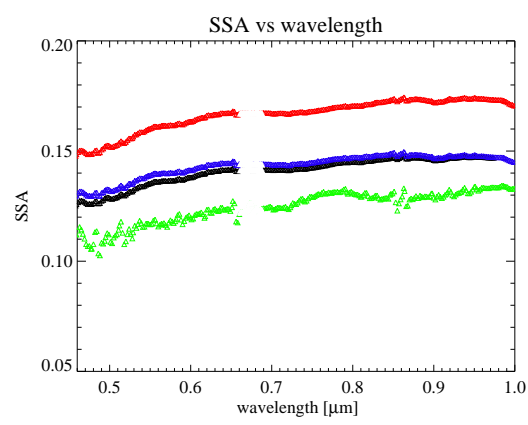

(a)

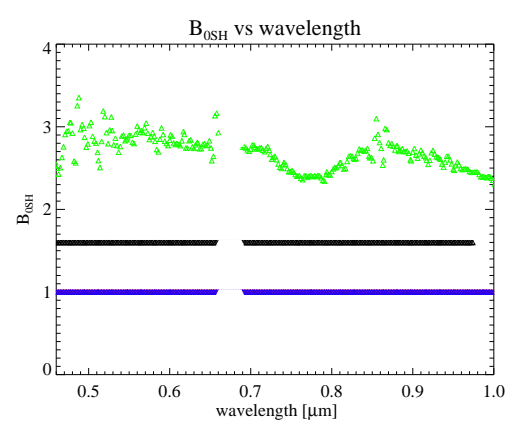

(c)

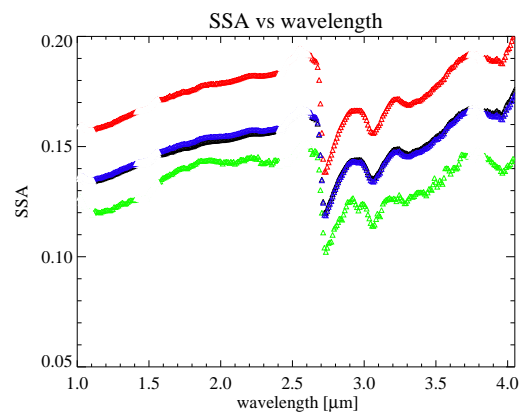

(a)

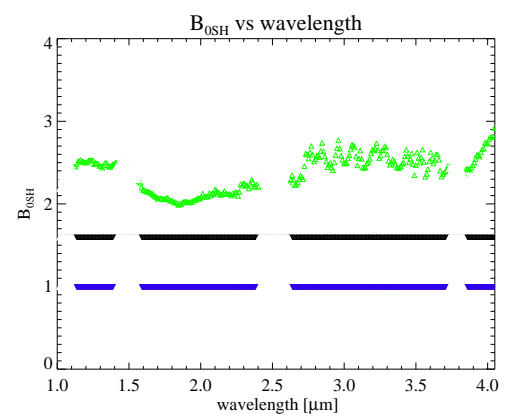

(c)

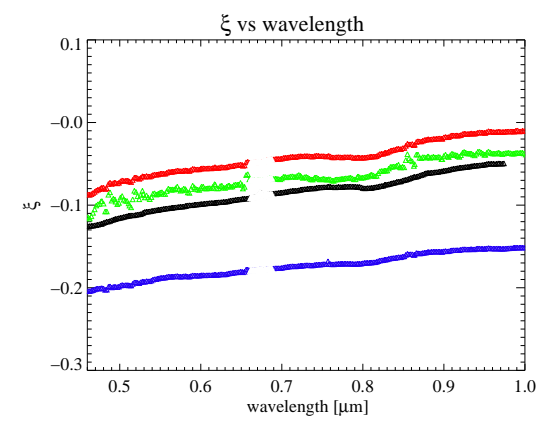

(b)

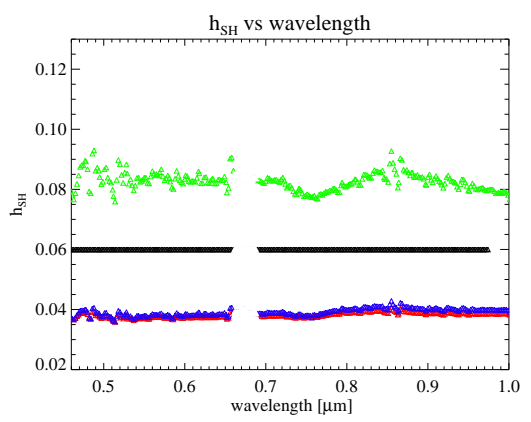

(d)

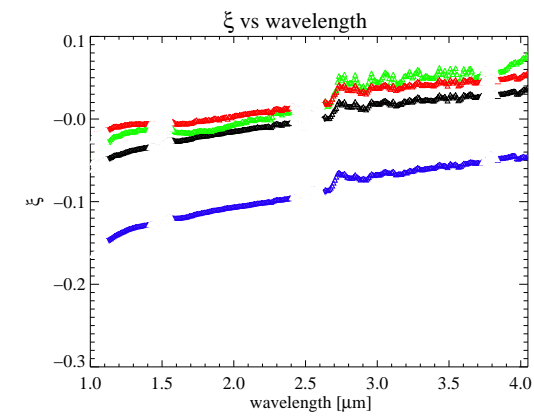

(b)

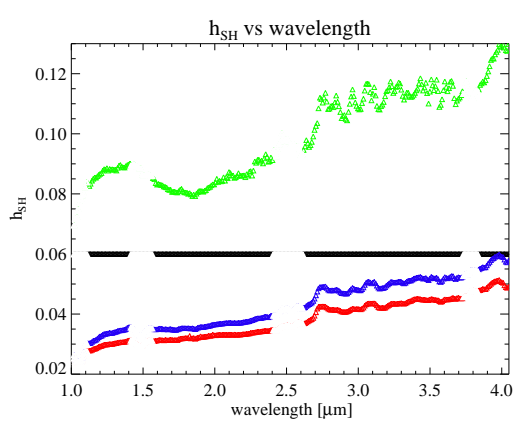

(d)
Fig. 6. Hapke's parameters at VIS wavelengths for different solutions of the Hapke model discussed in Sect. 3.3: S1 (black), S2 (green), S3 (red), and S4 (blue). (a) SSA, (b) $\xi$, (c) $B_{0 \mathrm{SH}}$, (S3 and $\mathrm{S} 4$ curves are superimposed) and $(d) h_{\mathrm{SH}}$. Gaps in the spectrum correspond to ordersorting filter positions and VIS-IR spectral junction of the instrument.

\subsubsection{Solution $\mathrm{S} 2$}

In Figs. 6 and 7, the retrieved Hapke model parameters (green symbols) for the $\mathrm{S} 2$ solution are shown, in comparison with the set S1 obtained in Ciarniello et al. (2017; black symbols) for VIS and IR wavelengths, respectively. For what concerns the two parameters $b$ and $c$, which determine the SPPF, we show the asymmetry parameter $\xi=-b c$, which describes the overall behavior of $p(\alpha)$, with a negative value of $\xi$ for a mostly backscattering SPPF and positive values in the case of a dominating forward-scattering contribution. In the case of the VIS channel (Fig. 6), the good phase angle coverage of Ceres' phase curve, in particular at low $\alpha$, allows us to partially limit the degeneration effects in Hapke's parameter retrieval (Helfenstein \& Shepard 2011). In particular, this can be noted when $\xi$ (Fig. 6b) and $h$ (Fig. 6d) are compared, the former being characterized by a globally monotonic increase with wavelength, while the latter remains fairly constant, indicating that the variations of the two parameters are not coupled. Moreover, the behavior of $\xi$ indicates that Ceres' SPPF is progressively less back-scattering 


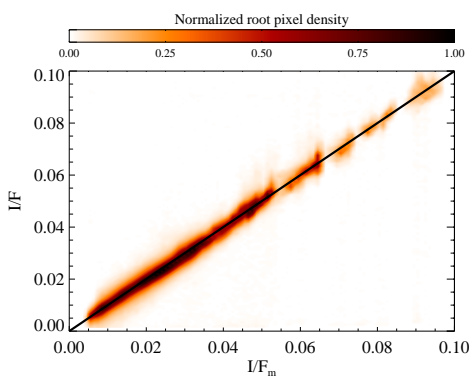

(a)

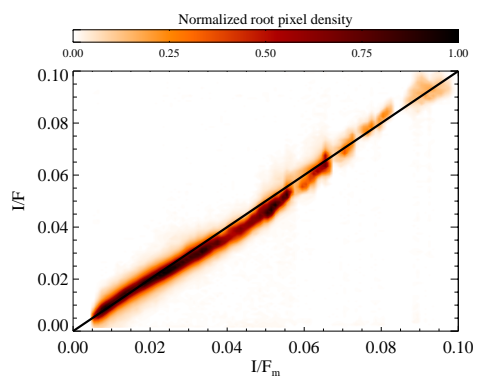

(b)

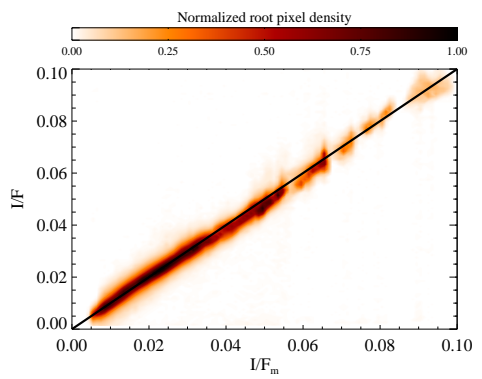

(c)

Fig. 8. Radiance factor $(I / F)$ as measured by VIR at $0.55 \mu \mathrm{m}$ against the modeled values $\left(I / F_{\mathrm{m}}\right)$ at the corresponding observation geometry, for different solutions of the Hapke model discussed in Sect. 3.3: S2 (a), S3 (b), and S4 (c). The black line in each plot indicates the ideal behavior $I / F=I / F_{\mathrm{m}}$. The color in the contour plots is proportional to the square root of the point density after normalization of the maximum value to unity.

with increasing wavelength, reflecting the global behavior of the phase curve described in Sect. 3. This also reinforces the idea that the reduction of back-scattering with wavelength is related to the single scattering process and to the optical properties of the single grains being reproduced within the SPPF. A comparison of the trends of $B_{0 \mathrm{SH}}(\mathrm{Fig} .6 \mathrm{c}), h_{\mathrm{SH}}$, and SSA reveals residual degeneration among these parameters, with $B_{0 \mathrm{SH}}$ and $h_{\mathrm{SH}}$ varying in correlated fashion while being anti-correlated with SSA. This suggests such variability is not necessarily physical, and instead may be the expression of the entanglement of the different parameters in modeling the phase curve, with larger SSA compensated by a less important OE term, with smaller amplitude $B_{0 \mathrm{SH}}$ and angular width $h_{\mathrm{SH}}$. The value of the SSA, which is between 0.10 and 0.13 , is consistent with a low albedo surface, but is lower with respect to the determination provided in $\mathrm{S} 1$. This is clearly explained by the fact that in S1, a constant value of $B_{0 \mathrm{SH}}=1.6$ was adopted from Helfenstein \& Veverka (1989), while in S2, $B_{0 \mathrm{SH}}$ is mostly found to be between 2 and 3, thus requiring a smaller SSA to match Ceres' phase curve and a modest adjustment of the SPPF.

Moving to the IR channel (Fig. 7), the lack of observations in the $1.6^{\circ}-7^{\circ}$ hinders a thorough determination of Hapke's parameters. In particular, the increase of $h_{\mathrm{SH}}$ (Fig. 7d) with wavelength is likely the effect of a degeneration with $\xi$ (Fig. 7b). Particular evidence for this is the fact that the derived trend of $h_{\mathrm{SH}}$ at the longest wavelength in the VIS seems to connect poorly to the behavior of $h_{\mathrm{SH}}$ at short wavelengths in the IR. Similar to what was observed in the VIS, $B_{0 \mathrm{SH}}$ (Fig. 7c) and $h$ (Fig. 7d) vary in correlated fashion below $2.5 \mu \mathrm{m}$, while a minor anti-correlated variation can be observed in the SSA (Fig. 7a). Also in this case, the SSA is smaller than S1, which is attributable to the large values of $B_{0 S H}$ in S2, again varying between 2 and 3 across the IR wavelength range. Apart from this, the spectrum of SSA shows the typical absorption features that are recognized on the surface of Ceres and are related to Mg-phyllosilicates $(2.72 \mu \mathrm{m})$, ammoniated phyllosilicates $(3.06 \mu \mathrm{m})$, and carbonates $(3.4$ and $3.9 \mu \mathrm{m})$ (De Sanctis et al. 2015), indicating that this parameter is clearly connected with the regolith's composition.

\subsubsection{Solutions S3 and S4}

According to Hapke's modeling of the OE, the parameter $B_{0 \mathrm{SH}}$, which describes the corresponding amplitude, represents the fraction of light that is scattered directly from the illuminated portion of the particle with respect to the total amount of light scattered at $0^{\circ}$ phase angle. Given this, its value can vary from zero, such as in the case of very transparent materials for which most of the light travels through the particles before being scattered, to one, for very opaque grains where the scattering process takes place mostly on the surface of the particles. However, the common application of Hapke's theory in the literature allows $B_{0 \mathrm{SH}}$ to exceed unity. Such a choice can be justified by assuming that roughness on the particle surface produces shadowing and single grains can have their own OE. Nonetheless, a rigorous application of the model would require these effects to be modeled directly within the SPPF. Values of $B_{0 S H}$ exceeding unity may then indicate that the SPPF contribution at small phase angles is modeled within the OE effect term $B_{\mathrm{SH}}(\alpha)$, possibly limiting the physical interpretation of the retrieved parameters.

Following this reasoning, a fit of Ceres' phase curve was performed imposing $B_{0 \mathrm{SH}} \leq 1$. We refer to this set of parameters as S3 (Figs. 6 and 7, red symbols). We note that for all the wavelengths, the solutions converge to the maximum value of the $\mathrm{OE}$ amplitude $B_{0 \mathrm{SH}}=1$. From a qualitative point of view, this is compatible with Ceres' low albedo and with the fact that its surface is dominated by opaque grains. It can also be noted that the smaller $B_{0 \mathrm{SH}}$ is partially compensated by a larger SSA with respect to $\mathrm{S} 1$ and $\mathrm{S} 2$. Also, the angular amplitude of the $\mathrm{OE} h_{\mathrm{SH}}$ is now smaller, but is still substantially constant in the VIS. As for the set of solutions S1 and S2, the SPPF is progressively less back-scattering with increasing wavelength, as indicated by the behavior of $\xi$, in particular for the VIS case. At IR wavelengths, also for S3, the physical interpretation of the corresponding results is complicated by the limited phase angle coverage in the $\mathrm{OE}$ region and the consequent degeneration of the different parameters.

To test the accuracy of the solutions $\mathrm{S} 2$ and $\mathrm{S} 3$, the $I / F$ at $0.55 \mu \mathrm{m}$ for each pixel acquired by VIR is compared to the modeled $I / F_{\mathrm{m}}$ at the same observation geometry (Fig. 8). In both cases, the modeled values are in good agreement with the measured ones, and the two quantities are linearly correlated (the correlation coefficient is $R=0.99$ for both cases). Nonetheless, the average relative error between the modeled and measured reflectance is slightly larger for S3 than for S2 (9\% against 7\%). In addition to this modest difference, the slightly lower accuracy of S3 is revealed in Fig. 8, where for reflectances of around 0.05 , a systematic deviation of $I / F$ versus $I / F_{\mathrm{m}}$ from the linear behavior can be noted. This difference in accuracy could be interpreted as the limited capability of the adopted form of the SPPF to correctly describe the real behavior of the regolith, in particular at low phase angles; this is compensated for by the $\mathrm{OE}$ term in $\mathrm{S} 2$, for which $B_{0 \mathrm{SH}}$ can exceed unity. We then 
apply a more flexible formulation of the SPPF, using a threeparameter Henyey-Greenstein expression (see also Clark et al. 1999), following the definition by Hapke (2012):

$$
\begin{aligned}
p(\alpha)= & \frac{1+c}{2} \frac{1-b_{1}^{2}}{\left(1-2 b_{1} \cos (\alpha)+b_{1}^{2}\right)^{3 / 2}} \\
& +\frac{1-c}{2} \frac{1-b_{2}^{2}}{\left(1+2 b_{2} \cos (\alpha)+b_{2}^{2}\right)^{3 / 2}} .
\end{aligned}
$$

In this case, the angular amplitude of the back-scattering and forward-scattering lobes are described by two separate parameters, $b_{1}$ and $b_{2}$, respectively. The asymmetry parameter is expressed as $\xi=-\frac{1+c}{2} b_{1}+\frac{1-c}{2} b_{2}$. The set of parameters, which we refer to as $\mathrm{S} 4$, derived by means of this approach, are again shown in Figs. 6 and 7 (blue symbols). At VIS wavelengths, the $\mathrm{OE}$ parameters are similar to $\mathrm{S} 3$, with the $\mathrm{OE}$ amplitude always reaching the maximum value of $B_{0 \mathrm{SH}}=1$ and the angular width $h_{\mathrm{SH}}$ which is mostly constant at $\approx 0.04$. Overall, the SSA is fairly similar to the result of S1, while the SPPF is now more back-scattering, with $\xi$ being significantly lower than the values obtained in the other solutions. Nonetheless, its spectral behavior still indicates a reduction in back-scattering with increasing wavelength. In the IR, as for the other set of parameters, degeneration of $h_{\mathrm{SH}}$ and $\xi$ can be noted, with a likely spurious variation of $h_{\mathrm{SH}}$ with wavelength, while the spectral properties of the SSA reflect the average Ceres composition.

The accuracy of this solution of the Hapke model (Fig. 8c) is compatible with the one obtained with $\mathrm{S} 2$, having a high correlation between $I / F$ and $I / F_{\mathrm{m}}(R=0.99)$ and an average error on the reflectance of the order of $7 \%$ at $0.55 \mu \mathrm{m}$. Nonetheless, it can be noted that a small residual $(\approx 5 \%)$ systematic deviation from the ideal behavior at $I / F=0.04-0.06$ is present. This reflects a less accurate match in the phase angle interval $10^{\circ}-25^{\circ}$, and indicates that a three-term SPPF is not able to fully compensate for the constraints imposed on the OE term. Nevertheless, given the overall good photometric accuracy of S4, and that the effect described above appears to be minor, it seems unnecessary to test more complex forms of the SPPF with a larger number of free parameters (e.g., by employing a Legendre polynomial expression).

\subsubsection{The surface roughness parameter}

Along with the SSA, the SPPF parameters, and the OE term, the average surface slope $\bar{\theta}$ has also been determined for all the solutions of the Hapke model discussed above. In Ciarniello et al. (2017), the obtained value was $\bar{\theta}=29^{\circ}{ }_{-6^{\circ}}$. Following the same approach, we derive $\bar{\theta}=29_{-5^{\circ}}^{\circ}$ for $\mathrm{S} 2, \bar{\theta}=31_{-6^{\circ}}^{\circ+8^{\circ}}$ for $\mathrm{S} 3$, and $\bar{\theta}=28^{\circ+4^{\circ}}$ for S4. Interestingly, $\bar{\theta}$ is compatible among the different solutions well within the estimated error bars, despite the significant differences already noted between the other Hapke model parameters. This indicates a limited correlation among parameters of the model describing very different parts of the phase curve, with the ones of the OE term being sensitive to the phase curve behavior at low phase angles, and $\bar{\theta}$ being mostly constrained by observations at large $\alpha$. This result further supports the possibility of providing a physical interpretation of Hapke's parameters.

\subsubsection{Angular width of the opposition effect, and regolith porosity}

Among the different solutions described in Sects. 3.3.1 and 3.3.2, $\mathrm{S} 4$ has the advantage of being obtained assuming that $B_{0 \mathrm{SH}}$ cannot exceed unity, as provided by Hapke's model. This allows us to attempt a physical interpretation of the angular width of the OE $h_{\mathrm{SH}}$, which according to SHOE theory (Hapke 2012) depends on the porosity of the investigated material. In this respect, we notice that a link between porosity and SHOE width has been evidenced by Stankevich et al. (1999) and Ciarniello et al. (2014) through Monte Carlo ray-tracing simulations in the geometric optics, while Helfenstein \& Shepard (2011) were able to provide good porosity estimates by means of Hapke's formalism applied to reflectance measurements on powder samples characterized by low and medium albedo. As discussed above, the overall spectral distribution of $h_{\mathrm{SH}}$ in the VIS, where a more robust determination of Hapke's parameters can be provided by means of the VIR dataset, is constant with wavelength and fully compatible with SHOE. In this regime, assuming that the surface is composed of equant particles of the same size and larger than the wavelength, $h_{\mathrm{SH}}$ can be connected to the filling factor $(\phi$, the fraction of volume occupied by particles) of the investigated material by the relation $h_{\mathrm{SH}}=-\frac{3}{8} \ln (1-\phi)$ (Hapke 1993). In the case of S4 with $h \approx 0.04$, this gives $\phi \approx 0.1$, corresponding to a porosity $P=1-\phi \approx 0.9$. This result is also confirmed with an independent characterization of the SHOE contribution obtained by comparing Ceres' phase curve to Monte Carlo ray-tracing simulations (see Appendix A).

Analysis. The high value of $P$ indicates a very porous surface and can be considered as an upper limit, given that according to Hapke's treatment of SHOE, a real grain size distribution which is not monodisperse can provide a similar OE peak for larger values of $\phi$. High values of porosity inferred from Hapke's modeling are not uncommon for planetary surfaces (Domingue et al. 2002; Hapke \& Sato 2016; Hasselmann et al. 2017). This result, at least from a qualitative point of view, is in good agreement with the low thermal inertia of Ceres, as derived from both ground-based observations (Müller \& Lagerros 1998; Chamberlain et al. 2009) and recent analysis of VIR data (Rognini et al. 2019), which may be compatible with a highly porous regolith. However, it must be considered that the thermophysical processes on a planetary surface are affected by the physical properties of the medium down to at least a few centimeters depth. Conversely, remote sensing observations at VIS wavelengths sample a much shallower surface layer. Given this, the porosity determination provided here may reflect the physical properties of the upper boundary of the medium involved in the thermophysical processes. A rough estimation of the typical thickness sensed by the OE observations can be provided by deriving the depth at which regolith particles contribute to SHOE. This can be computed considering that single scattering is the main contributor to the reflectance surge, thus implying that the maximum depth reached by light producing the OE is the depth at which the medium becomes optically thick. In the limit of low filling factor, the optical depth can be expressed as $\tau=n \sigma \Delta z$, where $n=\phi / v$ is the numerical density of the particles, $v=4 / 3 \pi R^{3}$ is the particle volume for a radius $R, \sigma=\pi R^{2}$ is the cross-section, and $\Delta z$ the depth in the material. The medium is optically thick when $\tau=1$, which gives $\Delta z=1 /(n \sigma)=\frac{4}{3} \frac{R}{\phi}$. A filling factor of 0.1 then yields $\Delta z \approx 13 R$, which assuming a grain size of $100 \mu \mathrm{m}$ (Raponi et al. 2019b) provides a depth of the order of $\approx 0.5 \mathrm{~mm}$, corresponding to the very first surface layers.

\subsection{Comparison with Hapke's parameters derived from Framing Camera observations}

In Table 2, we compare the different sets of parameters derived above with recent results from Hapke's modeling of FC data for 
Table 2. Comparison of Hapke's parameters for the surface of Ceres at visible wavelengths as derived from VIR (0.55 $\mu \mathrm{m})$ and FC observations.

\begin{tabular}{cccccc}
\hline \hline$B_{0 \mathrm{SH}}$ & $h_{\mathrm{SH}}$ & $\xi$ & $w$ & $\bar{\theta}[\mathrm{deg}]$ & Reference \\
\hline$(1.6)$ & $(0.06)$ & $-0.11 \pm 0.08$ & $0.14 \pm 0.02$ & $29 \pm 6$ & S1, Ciarniello et al. (2017) \\
$2.9_{-0.2}^{+0.1}$ & $0.084_{-0.004}^{+0.003}$ & $-0.085 \pm 0.08$ & $0.12_{-0.01}^{+0.02}$ & $29 \pm 5$ & S2, this work \\
1 & $0.037_{-0.001}^{+0.002}$ & $-0.062 \pm 0.09$ & $0.16_{-0.02}^{+0.04}$ & $31_{-6}^{+8}$ & S3, this work \\
1 & $0.038_{-0.01}^{+0.02}$ & $-0.19 \pm 0.04$ & $0.13 \pm 0.01$ & $28_{-4}^{+4}$ & S4, this work \\
1.6 & 0.054 & -0.11 & 0.116 & 23 & ${\text { Schröder et al. }(2018)^{(a)}}^{(b)}$ \\
$(1.6)$ & $(0.06)$ & -0.03 & $0.143_{-0.04}^{+0.05}$ & $19 \pm 6$ & ${\text { Li et al. }(2019)^{(b)}}$
\end{tabular}

Notes. Values in parentheses are from Helfenstein \& Veverka (1989). For the results of Ciarniello et al. (2017) and this work, the error on the parameters was computed starting from the range of confidence obtained on the roughness average slope $\bar{\theta}$ (see Ciarniello et al. 2017). For each value of the roughness in this interval, the fitting procedure converges to a different set of parameters, and their variability is assumed as the associated uncertainty. ${ }^{(a)}$ Hapke's model adopting a double-parameter Henyey-Greenstein SPPF (Case C solution) with FC Clear Filter data. Errors on the parameters are of the order of unity on the last digit. ${ }^{(b)}$ Hapke's model adopting a double-parameter Henyey-Greenstein SPPF (2pHG) at $555 \mathrm{~nm}$ (FC F2 filter).

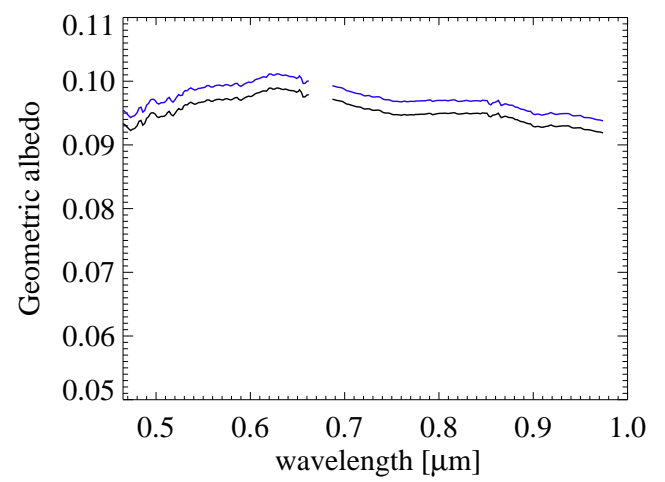

(a)

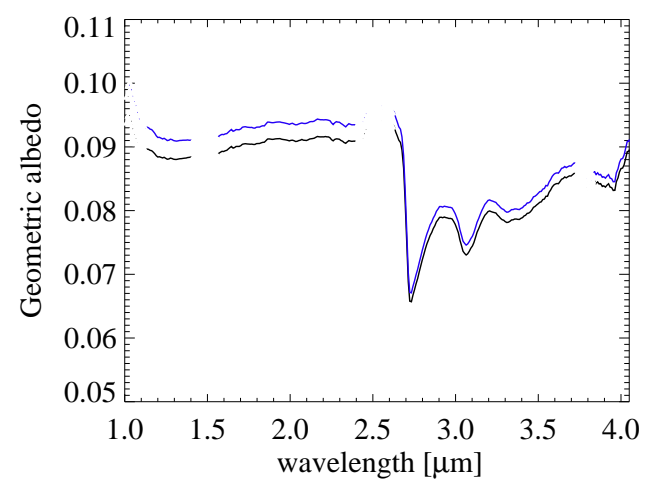

(b)
Fig. 9. Geometric albedo of Ceres at VIS $(a)$ and IR $(b)$ wavelengths. Black curves are from S2 and blue curves from S4. Missing parts of the spectrum correspond to the order-sorting filter positions and VIS-IR spectral junction of the instrument.
Ceres at visible wavelengths from Schröder et al. (2018) and $\mathrm{Li}$ et al. (2019). It can be noted that there is a good overall agreement among the different sets of solutions. In particular, the single scattering albedo's central values are all approximately comprised in the $0.12-0.16$ range, while the central values of the asymmetry parameters indicate SPPFs with a modest back-scattering behavior. The lowest asymmetry parameter is exhibited by $\mathrm{S} 4$, which was derived from a three-parameter implementation of the SPPF, and may not be directly comparable with the other values. The roughness parameters of the different solutions are compatible within the uncertainty ranges, although VIR estimations give typically larger central values. This could be due to the systematically lower spatial resolution of VIR observations with respect to FC data, which introduces a larger contribution from unresolved shadows if the surface morphology of Ceres is not fractal at these spatial scales. Finally, the largest differences are encountered in the determination of the OE parameters. In this case, a direct comparison with the Hapke models from FC observations considered here can only be performed with the S2 solution, where no restrictions on the $\mathrm{OE}$ amplitude $B_{0 \mathrm{SH}}$ were imposed. Here we retrieve the largest values of $B_{0 \mathrm{SH}}$ and $h_{\mathrm{SH}}$, when compared with the values from Helfenstein \& Veverka (1989) and Schröder et al. (2018). It must also be noted that Schröder et al. (2018), who investigated different solutions of the Hapke model, report different values of $\mathrm{OE}$ parameters, with Case $\mathrm{A}$ in their paper providing numbers compatible with ours $\left(B_{0 \mathrm{SH}}=3.1\right.$ and $\left.h_{\mathrm{SH}}=0.081\right)$. This suggests that the determination of the OE amplitude and angular width when $B_{0 \mathrm{SH}}$ is allowed to exceed unity can be significantly affected by the interplay and entanglement of the different parameters in Hapke's model. Furthermore, small variations in the estimated SSA, SPPF, and roughness may produce major variations of the OE parameters. Nonetheless, the similarity of our results with Case A of Schröder et al. (2018) likely reflects the similarity in OE surge morphology observed by the VIR and FC instruments.

\subsection{Geometric albedo}

Using the OE data, we can provide an updated estimation of the geometric albedo of the surface of Ceres with respect to Ciarniello et al. (2017). To this aim, we employ Hapke's model with the set of parameters providing the most accurate photometric results: S2 and S4. The derived geometric albedo spectra $^{6}$ are reported in Fig. 9. The results obtained with the two sets of parameters show a systematic difference, with that from S2 slightly shifted towards lower values by $2 \%$. This is due to the uncertainty associated with the two photometric models along with the different approach adopted to derive Hapke's parameters between the two solutions. In S2, the possibility to deal with an opposition effect amplitude parameter $B_{0 \mathrm{SH}}$ greater than 1 allows for a larger flexibility of the model in the OE region with respect to $\mathrm{S} 4$. Given the limited surface coverage of the OE observations, in particular near the opposition, the local

$\overline{6}$ Here we are not accounting for the effect induced by the finite angular size of the Sun. In this respect, our values could be overestimated by $\approx 0.5 \%$, which is much smaller than the typical uncertainty on the derived geometric albedo. 
variability of the albedo of the sampled regions can be reflected in the derived geometric albedo. Such a possibility is in principle limited in $\mathrm{S} 4$ where it is imposed that $B_{0 \mathrm{SH}} \leq 1$, which may provide a result that is more representative of the average photometric properties of Ceres. With a conservative approach, we take the average of the two estimations as the reference value for the geometric albedo of Ceres and assume an error of $7 \%$, which is the typical accuracy of the two photometric models (see Sect. 3.3) in reproducing the resolved data and should represent an upper limit on the real uncertainty associated to the geometric albedo. This provides a geometric albedo of $0.098 \pm 0.007$ at the wavelength of $0.55 \mu \mathrm{m}$. This value is still compatible with the previous estimation given in Ciarniello et al. (2017) from VIR data not including opposition observations (0.094 \pm 0.007$)$, where OE parameters were assumed from Helfenstein \& Veverka (1989), and with the results from Reddy et al. (2015), Schröder et al. (2018), and Li et al. (2019) at VIS wavelengths.

\section{Disk-resolved opposition effect properties in the VIS range}

The set of VIR OE observations provided the possibility to observe part of the surface of Ceres with multiple passages and with varying phase angles. This allowed us to derive phase curves in the interval $0^{\circ}-7^{\circ}$ for different positions across the surface, with the aim to search for spatial variability of the $\mathrm{OE}$ properties ${ }^{7}$. As described in Sect. 3, in order to account for the photometric effects related to incidence and emission angle variation, $I / F$ as measured by VIR was divided by the corresponding disk-function $D$, assuming the roughness parameter from $\mathrm{S} 2$, $\bar{\theta}=29^{\circ}$. To ensure a sufficient number of pixels and the broadest possible coverage of the OE part of the phase curve for each position, VIR observations were mapped according to a $2^{\circ} \times 2^{\circ}$ latitude-longitude grid. However, given the limited number of acquisitions for the VIS channel, only a small portion of the surface can be investigated, roughly comprised between longitudes $175^{\circ}-260^{\circ}$ and latitudes $-25^{\circ}$ to $50^{\circ}$ (Fig. 1), while for the IR channel, the availability of only a single observation prevents us from applying the same method. The phase curve in each position is fitted with the empirical function

$\frac{I / F}{D}=A_{0} \exp (-v \alpha)$

with an approach similar to Schröder et al. (2018), where $A_{0}$ is the normal albedo of the surface, $v$ is a morphological parameter which is related to the OE angular width, and $\alpha$ is expressed in degrees (Fig. 10). To provide a proper sampling of the phase curve, only positions on the surface with more than five observations, a minimum phase angle $\alpha_{\min }<1.2^{\circ}$, and with a difference between the maximum and minimum phase angle $\alpha_{\max }-\alpha_{\min }>4^{\circ}$ were selected. Moreover, in order to provide the same weight to the different phase angles, the observations

\footnotetext{
7 Recently, Rousseau et al. (2019) provided evidence that VIR VIS responsivity is affected by the CCD temperature, which introduces a progressive blueing of the spectrum. This effect averages out when the global dataset is used to derive Ceres' spectrophotometric properties, as for the analysis reported above. Nonetheless, it can be significant when mapping of the observations is performed and, because of limited statistics, different regions are sampled with different CCD temperatures To overcome this issue, in this section the $\mathrm{OE}$ data have been corrected for the CCD temperature effect following the method described in Rousseau et al. (2019), to which the reader is referred for further details, and that provides a correction accurate at a roughly $4 \%$ level.
}

Table 3. Average values of $A_{0}$ and $v$ across the mapped portion of the surface of Ceres (see Fig. 11).

\begin{tabular}{ccc}
\hline \hline Wavelength $[\mu \mathrm{m}]$ & $A_{0}$ & $v$ \\
\hline 0.465 & $0.0943 \pm 0.0024$ & $0.0607 \pm 0.0025$ \\
0.550 & $0.0964 \pm 0.0021$ & $0.0604 \pm 0.0027$ \\
0.649 & $0.0975 \pm 0.0019$ & $0.0600 \pm 0.0026$ \\
0.700 & $0.0956 \pm 0.0017$ & $0.0601 \pm 0.0026$ \\
0.749 & $0.0937 \pm 0.0017$ & $0.0612 \pm 0.0025$ \\
0.800 & $0.0937 \pm 0.0017$ & $0.0591 \pm 0.0025$ \\
0.900 & $0.0907 \pm 0.0016$ & $0.0591 \pm 0.0026$ \\
\hline
\end{tabular}

Notes. Errors are computed as the standard deviation of the distributions after sigma-clipping.

were resampled in bins of $0.05^{\circ}$ in width, averaging pixels occurring in the same bin.

Analysis. In Fig. 11, the fitted values of $A_{0}$ and $v$ are mapped on the surface of Ceres for different selected wavelengths. It can be noted that, although albedo variability can be revealed in $A_{0}$ maps, in particular in the vicinity of surface features like Occator, Azacca, Nawish, Heneb and Lociyo craters, this is not followed by a similar distribution of $v$, confirming, also at local scale, the general finding of a lack of correlation between albedo and $\mathrm{OE}$ width in the $0^{\circ}-7^{\circ}$ phase angle interval. On the other hand, the variability of $v$ across the surface seems to be partially correlated with the phase angle coverage (we highlight the smaller values at latitudes below $-5^{\circ}$ and longitudes greater than $210^{\circ}$, which correspond to a sharp increase in the $\alpha_{\min }$ map and to smaller values in the $\alpha_{\max }-\alpha_{\min }$ map). Nonetheless, intrinsic variability of $v$ can be seen, as shown for example at longitude $200^{\circ}-220^{\circ}$ and latitude $-5^{\circ}$ to $20^{\circ}$, where a distribution of larger values with respect to the surrounding terrains is found, which appears to be uncorrelated with phase angle coverage or albedo. Such spatial variability of $v$ is similar across the VIS spectral range, suggesting it is the expression of slightly different physical properties of the surface in terms of grain size and/or porosity. Average values of $A_{0}$ and $v$ are indicated for different wavelengths in Table 3. Again, $v$ does not show significant correlation with wavelength or albedo, confirming a similar finding by Schröder et al. (2018). Such behavior is compatible with a main contribution of SHOE, which is expected to dominate in this phase angle interval.

Schröder et al. (2018) showed evidence of correlation between the $\mathrm{OE}$ slope and albedo in correspondence with the ejecta of Azacca crater for $\alpha<0.6^{\circ}$, making this area a possible candidate for $\mathrm{CBOE}$; unfortunately, we cannot confirm this finding, given the sparse coverage for such small phase angles in the VIR dataset and because of the modest increase of signal expected in this phase angle interval $\left(\approx 5 \%\right.$ for $\alpha$ from $0.6^{\circ}$ to $\left.0^{\circ}\right)$, which is still comparable with our calibration residuals (Fig. 10).

\section{The opposition effect of Ceres compared to that of asteroids}

The majority of asteroid observations at low phase angles are performed using ground-based telescopes and refer to the integrated full-disk brightness of the target. In order to compare the opposition effect properties of Ceres as derived by VIR with published literature, we compute a $V$-band integrated magnitude phase curve starting from the measured reflectance from resolved observations using the same approach as described in 


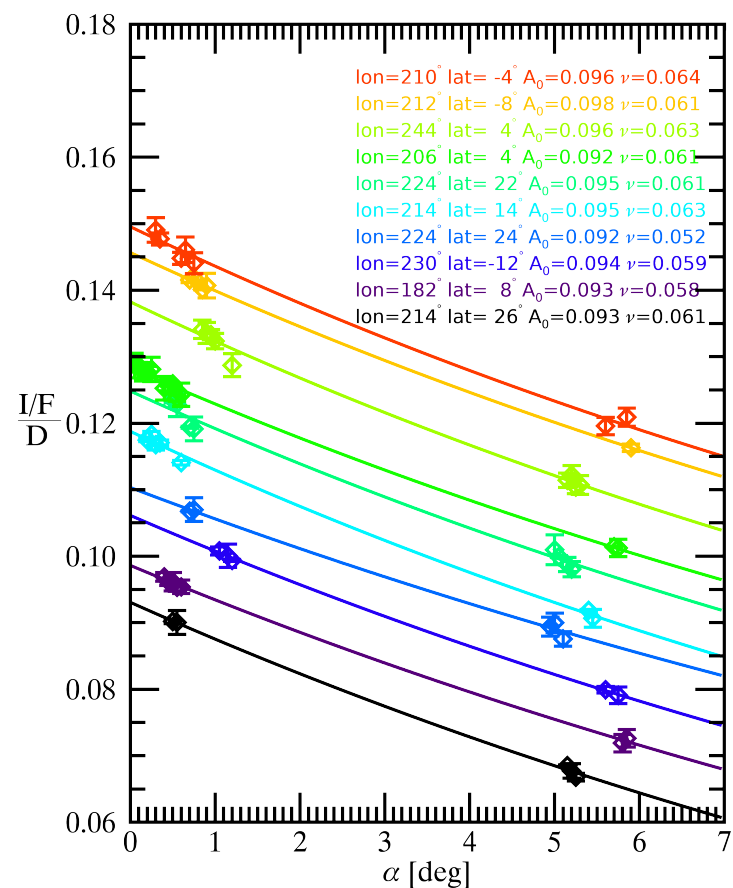

(a)

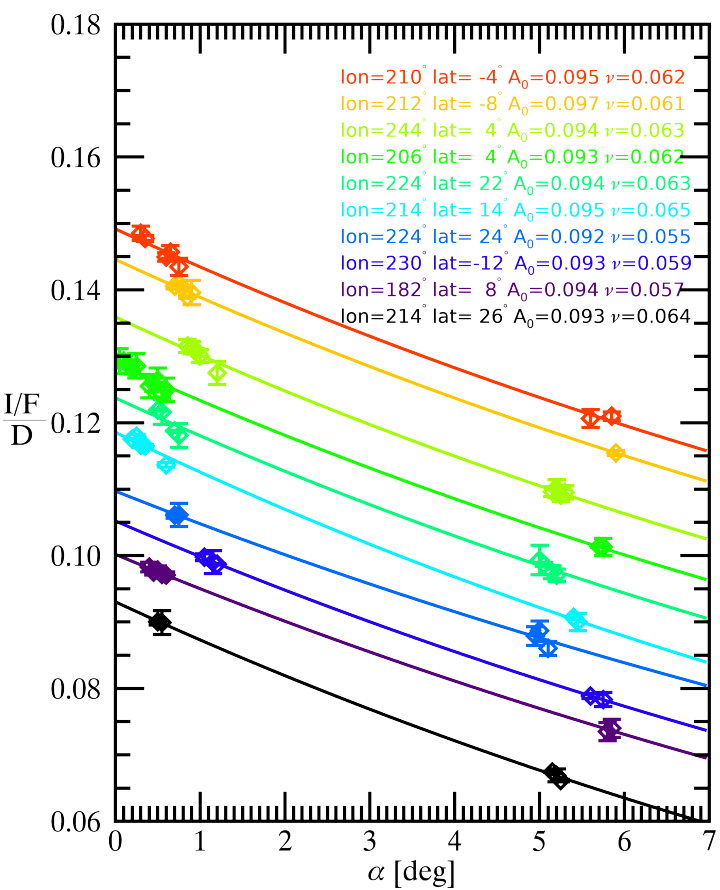

(c)

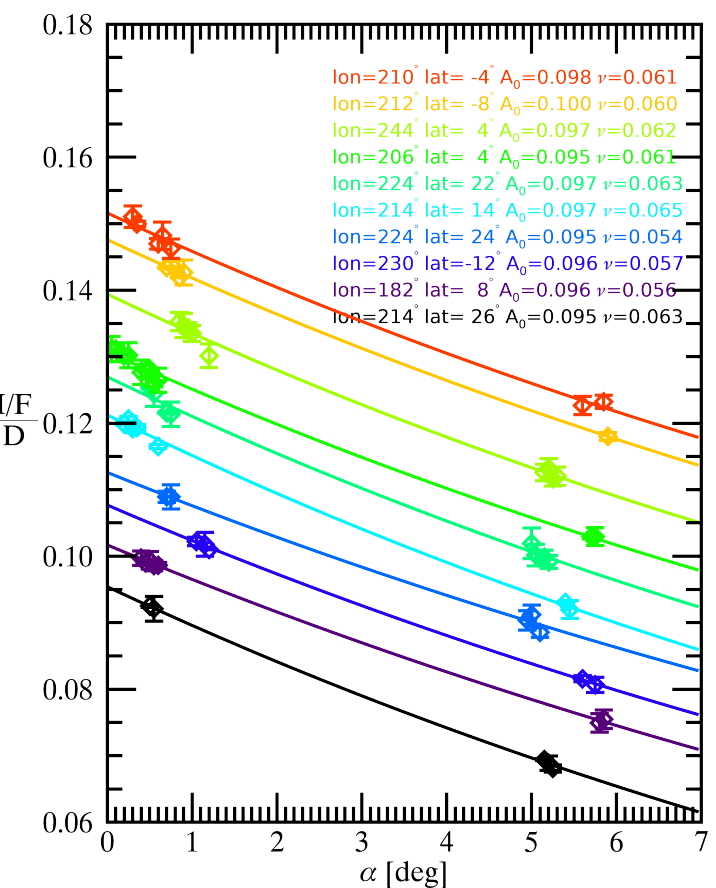

(b)

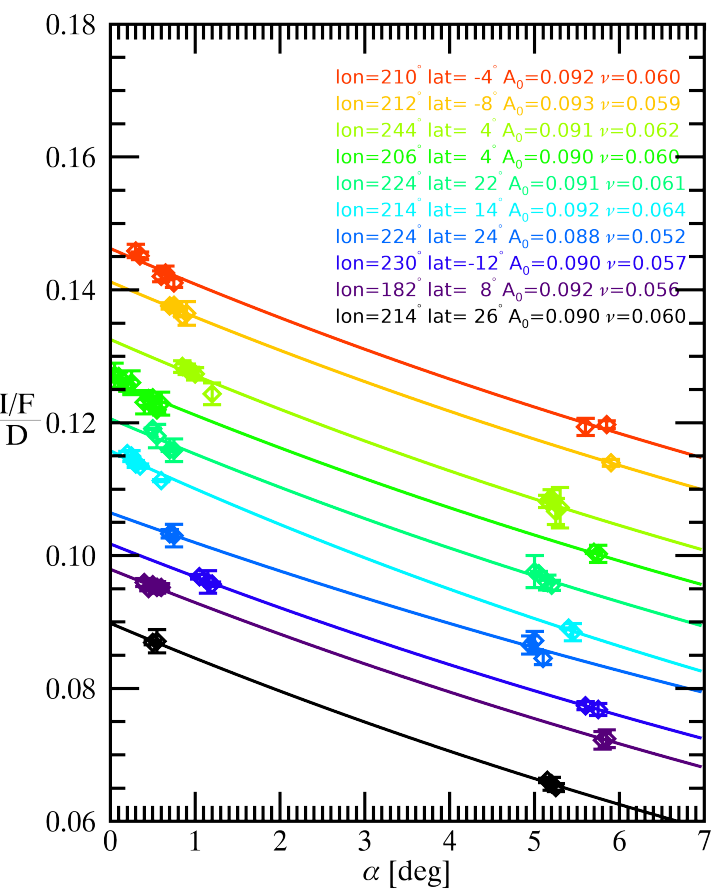

(d)

Fig. 10. Examples of phase curves at different wavelengths $(a: 0.465 \mu \mathrm{m} ; b: 0.550 \mu \mathrm{m} ; c: 0.749 \mu \mathrm{m} ; d: 0.900 \mu \mathrm{m})$ and different positions on the surface of Ceres as derived from OE data. Diamonds correspond to VIR observations and solid lines are fits obtained with Eq. (4). For each curve, the longitude and latitude of the corresponding position on the surface are indicated, along with the values of the fitted parameters. Curves are offset by 0.006 for clarity.

Ciarniello et al. (2017) (Fig. 12), and extending the phase angle coverage down to opposition, with a phase angle sampling of $0.2^{\circ}$ for $\alpha<7^{\circ}$. It can be noted that the derived phase curve shows an approximately linear behavior at larger phase angles down to $\approx 7^{\circ}$, followed by a steeper increase of brightness in the OE region. Such behavior is common to different asteroids, as reported by Belskaya \& Shevchenko (2000), with a certain variability in terms of amplitude and angular width of the OE surge. Here we compare the OE properties of Ceres' magnitude phase curve with those derived for the different asteroids investigated in Belskaya \& Shevchenko (2000) at $\alpha<25^{\circ}$, where the authors apply two parameters to describe the brightness surge at small phase angles: ratio of intensities $I\left(0.3^{\circ}\right) / I\left(5^{\circ}\right)$, namely the ratio of the integrated radiances at $\alpha=0.3^{\circ}$ and $\alpha=5^{\circ}$; and 


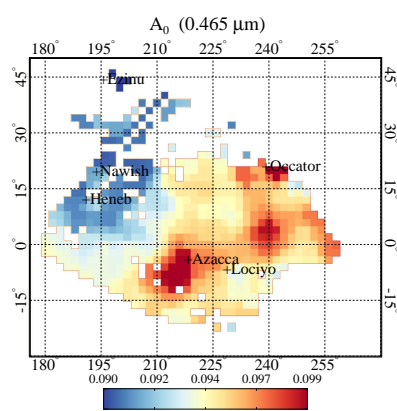

(a)

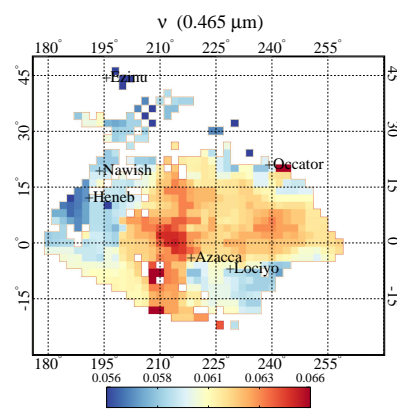

(e)

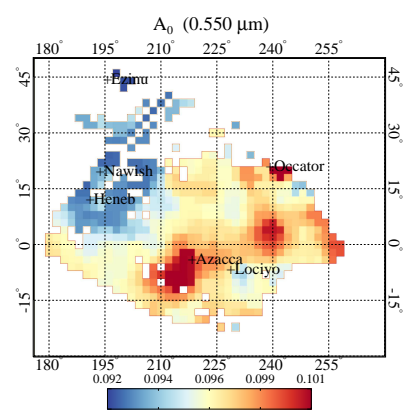

(b)

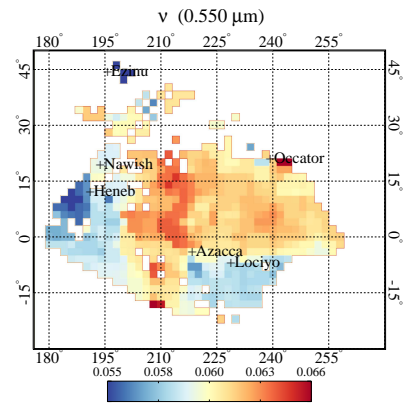

(f)

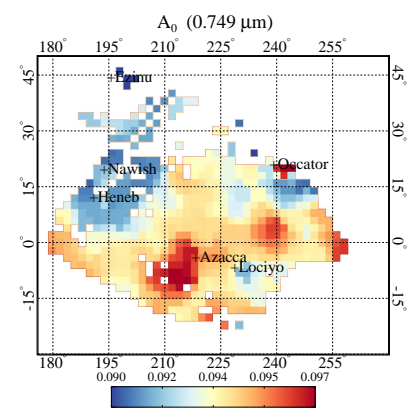

(c)

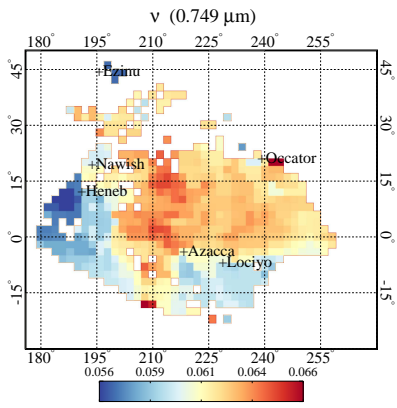

(g)

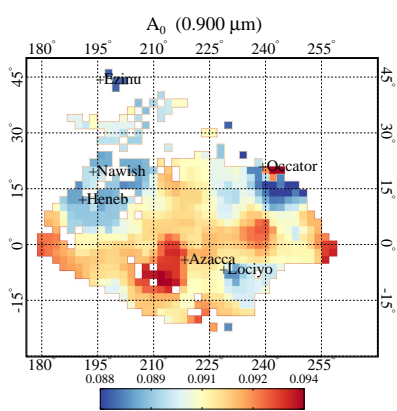

(d)

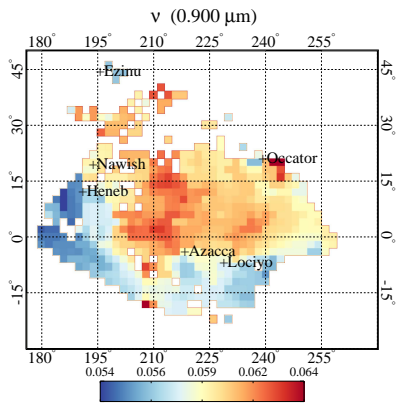

(h)

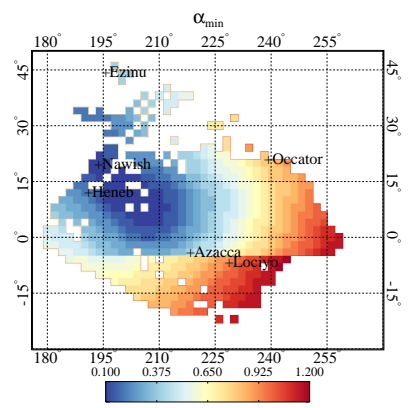

(i)

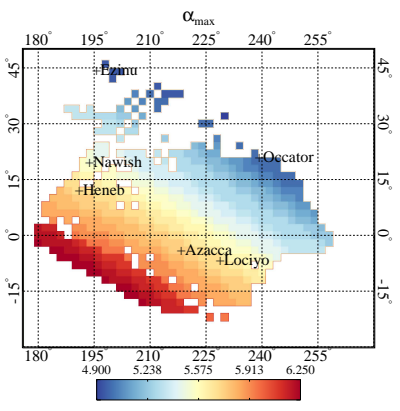

(j)

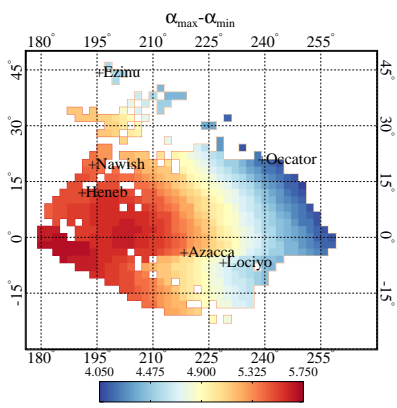

(k)

Fig. 11. $A_{0}(a-d)$ and $v(e-h)$ maps at different wavelengths. Also maps of $\alpha_{\min }, \alpha_{\max }$ and $\alpha_{\max }-\alpha_{\min }$ are shown $(i-k)$ for reference. A $3 X 3$ median filter has been applied to $A_{0}$ and $v$ maps to reduce local spurious variability. Color stretching is set to encompass values within $2 \sigma$ from the average of each parameter (after sigma clipping), and slightly differs from wavelength to wavelength. We note that values of $A_{0}$ in Occator's bright spots can be largely out of scale.

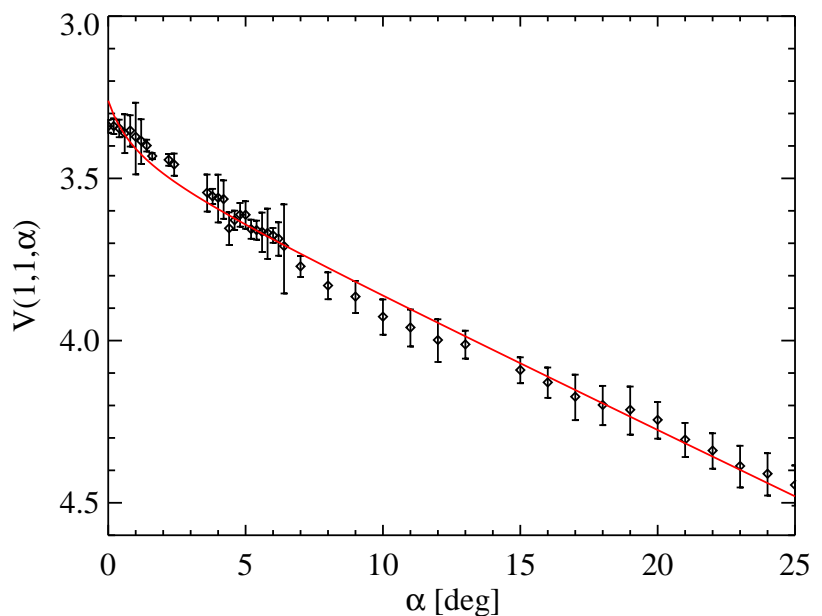

Fig. 12. Ceres' $V$-band magnitude phase curve, as derived from VIR observations. The red line is a fit in the phase angle range $0^{\circ}-25^{\circ}$ by means of the approximating function from Shevchenko et al. (1997). amplitude of OE, that is, the difference between the magnitude at $0.3^{\circ}$ and the corresponding extrapolated value at the same phase angle of the linear part of the phase curve outside the OE region.

To derive the amplitude of OE, following Belskaya \& Shevchenko (2000), we fit Ceres' magnitude phase curve with the approximating function from Shevchenko et al. (1997), which expresses the magnitude of the target as the sum of two terms, one accounting for the linear part of the phase curve and one depending on the amplitude of OE (Fig. 12). From the VIR data we obtain $I\left(0.3^{\circ}\right) / I\left(5^{\circ}\right)=1.33 \pm 0.05$ and amplitude of the $\mathrm{OE}=0.22 \pm 0.03$. These values are reported in Fig. 13, along with the ones derived for different asteroids investigated in Belskaya \& Shevchenko (2000) and for Ceres by Schröder et al. (2018), as a function of the geometric albedo $p_{V}$. From our study, we have $p_{V}=0.097 \pm 0.007$, after weighing with the solar spectrum and integration across the $V$-band of the average of the two estimations reported in Sect. 3.5. In the study of Belskaya \& Shevchenko (2000), the values of intensity ratio and amplitude of the OE for the different asteroidal types follow an arch-shaped distribution as a function of geometric albedo, with 


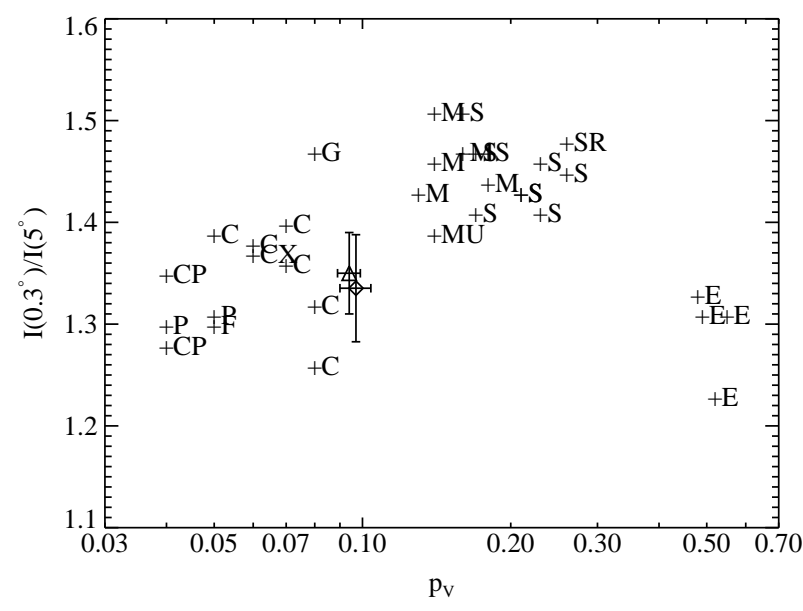

(a)

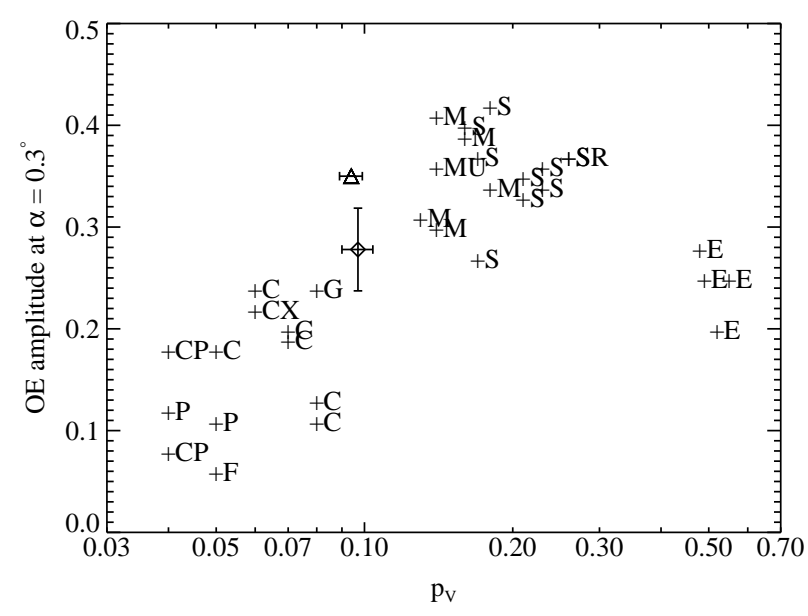

(b)

Fig. 13. Ratio of intensities $(a)$ and amplitude of the $\mathrm{OE}(b)$ as a function of geometric albedo for different asteroids from Belskaya \& Shevchenko (2000; crosses) and Ceres from this work (diamond) and from Schröder et al. (2018; triangle). Letters indicate the asteroid type according to Tholen (1989). In Schröder et al. (2018), the enhancement factor is reported, which we converted into the corresponding amplitude of the OE.

the darkest bodies exhibiting less pronounced OE surges, which progressively increase in amplitude going towards intermediate albedos reaching a maximum around $p_{V} \approx 0.2$, and then reducing again for the brightest objects. This trend is explained as the effect of the balance between SHOE and CBOE, with the former dominating on low albedo surfaces, where only single scattering is important, and the second being more effective on bright surfaces, where multiple scattering takes place. At intermediate albedos, the simultaneous contribution of the two mechanisms can produce a maximum in the $\mathrm{OE}$ amplitude. The values we obtained for Ceres align very well along the trend described above, showing OE properties in line with the ones expected for dark objects, and compatible with $\mathrm{C}$ class asteroids. This finding extends the previous results of Ciarniello et al. (2017) and Longobardo et al. (2019), which indicated C-type asteroids as a good match to Ceres' photometric properties, to the opposition effect region. A similar result was reported by Schröder et al. (2018), using FC data. The value of intensity ratio for Ceres found by these latter authors is in perfect agreement with our estimation, while their amplitude of $\mathrm{OE}$ is slightly larger, falling in the range of values mostly populated by asteroids with intermediate albedos, like M- and S-types.

\section{Summary and discussion}

Using VIR-Dawn observations of Ceres at low phase angles (Fig. 1) performed during XMO4 phase we characterized the $\mathrm{OE}$ effect on the dwarf planet and extended the spectrophotometric investigation of Ciarniello et al. (2017) to the broad $\alpha \approx 0^{\circ}-132^{\circ}$ interval (Fig. 2).

The phase curve for Ceres as derived from VIR shows a progressive decrease in the back-scattering behavior with wavelength across the VIS-IR spectral range (Fig. 3). The reduction in back-scattering is larger at VIS wavelengths (Fig. 4), suggesting a relation to roughness at submicron scale hosted on the $\approx 100 \mu \mathrm{m}$-sized regolith (Raponi et al. 2019b). Conversely, at low phase angles $\left(\alpha<10^{\circ}\right)$, the shape of the phase curve for Ceres is fairly constant in the VIS range, and shows only minor variability in the IR $\left(\alpha<2^{\circ}\right)$ (Fig. 5). For the latter case, the availability of only one IR observation prevents us from drawing firm conclusions on the nature of these variations, which could be the effect of surface spatial variability, since in a single acquisition, different phase angles sample different positions on the surface.

The phase curve for Ceres was modeled by means of Hapke's theory. Different implementations were tested by assuming: (1) a two-parameter Henyey-Greenstein SPPF and a free-toexceed-unity opposition effect amplitude $B_{0 \mathrm{SH}}(\mathrm{S} 2)$; (2) a twoparameter Henyey-Greenstein SPPF and $B_{0 \mathrm{SH}} \leq 1$ (S3); and (3) a three-parameter Henyey-Greenstein SPPF and $B_{0 \mathrm{SH}} \leq 1$ (S4). Solutions S2 and S4 provided the most accurate results (accuracy at VIS wavelengths of the order of 7\%). Among these two, S4 allowed us to attempt a physical interpretation of the $\mathrm{OE}$ angular width parameter $h_{\mathrm{SH}}$ in terms of porosity of the regolith, under the assumption that $\mathrm{SH}$ is the main mechanism producing the opposition surge. In particular, the fairly constant value of $h_{\mathrm{SH}} \approx 0.04$ at VIS wavelengths would correspond to a very porous material with $P \approx 0.9$. Such high porosity, which we further confirmed by comparison of Ceres' phase curve with Monte Carlo ray-tracing simulations (Fig. A.2), can contribute to producing the low thermal inertia of Ceres (Müller \& Lagerros 1998; Chamberlain et al. 2009; Rognini et al. 2019).

By means of OE observations and Hapke's modeling, we estimated Ceres' geometric albedo across the whole investigated spectral range $(0.465-4.05 \mu \mathrm{m}$; Fig. 9), providing a value of $0.098 \pm 0.007$ at $0.55 \mu \mathrm{m}(0.097 \pm 0.007$ when integrated across the $V$-band) in good agreement with results in the VIS range from Reddy et al. (2015), Schröder et al. (2018), and Li et al. (2019).

With the aim of characterizing the OE variability across the part of the surface sampled by the low-phase-angle observations, the reflectance surge angular width was mapped. This latter was derived by fitting an empirical exponential model to VIR VIS data in the $0^{\circ}-7^{\circ}$ phase angle range (Fig. 10). No evident correlation was found between the spatial distribution of the $\mathrm{OE}$ angular width and surface albedo. In addition, the average OE angular width is fairly constant across the VIS range and does not correlate with the average albedo at different wavelengths (Table 3). Nonetheless, spatial variability of $\mathrm{OE}$ angular width can be recognized, suggesting local variability of physical properties such as regolith porosity and grain size distribution (Fig. 11).

Finally, the OE properties of Ceres have been compared with the ones of a sample of asteroids analyzed in Belskaya \& Shevchenko (2000) as a function of the VIS geometric albedo 
by means of two parameters, the intensity ratio and the $\mathrm{OE}$ amplitude. The values found for Ceres appear to be typical of a low-albedo object exhibiting OE properties compatible with C-type asteroids (Fig. 13).

A major goal of this study was to define the mechanism producing the $\mathrm{OE}$ on Ceres. The lack of significant variability of the phase curve shape at small phase angles both with wavelength and albedo, as would be expected from theoretical prediction of CBOE (Mishchenko 1992) for silicatic surfaces, would be compatible with SHOE being the dominant process at work. This idea is also reinforced by the measured shape of the phase curve in the OE region, where no sharp surge at very small phase angles $\left(<2^{\circ}\right)-$ a phenomenon typically attributed to CBOE - can be recognized. Based on this, we attempted to interpret $\mathrm{OE}$ width of Ceres in terms of porosity, under the SHOE assumption. However, following the line of argument provided in Schröder et al. (2018), we want to mention that these criteria, and in particular the angular width wavelength dependence, may not be sufficient to definitely rule out the contribution of CBOE on Ceres and, in general, on a planetary surface. In this respect, several laboratory measurements of OE on powders (Nelson et al. 2002; Shkuratov et al. 2004; Kaasalainen et al. 2005) failed to reveal significant variability with wavelength for samples exhibiting sharp opposition surges. This would imply that if $\mathrm{CB}$ is responsible for the $\mathrm{OE}$ in these experiments, the scattering process in a closepacked medium is possibly more complex than that described by the yet physically rigorous Mishchenko (1992) theory. Furthermore, evidence of wavelength dependence of the OE width has been provided only in a few cases from remote sensing observations (see a complete list of references in Schröder et al. 2018). A more robust test to characterize the presence of $\mathrm{CBOE}$ on Ceres would rely on polarization measurements, which could not be performed by the Dawn experiments. In particular, the occurrence of a narrow peak in negative polarization at small phase angles $\left(\alpha<2^{\circ}\right.$, Rosenbush et al. 2006) could be indicative of CB (Shkuratov 1989; Muinonen 1990), but unfortunately the available ground-based measurements of the polarization phase curve for Ceres do not cover the very small phase angle region with sufficient angular resolution (Devogèle et al. 2018).

Acknowledgements. VIR is funded by the Italian Space Agency-ASI and was developed under the leadership of INAF-Istituto di Astrofisica e Planetologia Spaziali, Rome-Italy (ASI-INAF n. I/004/12/0). The instrument was built by Selex-Galileo, Florence-Italy. The authors acknowledge the support of the Dawn Science, Instrument, and Operations Teams. We thank Sharon Uy (UCLA, USA) for manuscript editing. We also thank the editor for his suggestions and Paul Helfenstein for the insightful review, which improved the paper.

\section{References}

Acton, C. H. 1996, Planet. Space Sci., 44, 65

Ammannito, E., De Sanctis, M. C., Ciarniello, M., et al. 2016, Science, 353, aaf 4279

Belskaya, I., \& Shevchenko, V. 2000, Icarus, 147, 94

Buratti, B. J., Hillier, J. K., \& Wang, M. 1996, Icarus, 124, 490

Carrozzo, F. G., Raponi, A., De Sanctis, M. C., et al. 2016, Rev. Sci. Instr., 87, 124501

Chamberlain, M. A., Lovell, A. J., \& Sykes, M. V. 2009, Icarus, 202, 487

Ciarniello, M., Capaccioni, F., Filacchione, G., et al. 2011, Icarus, 214, 541

Ciarniello, M., Capaccioni, F., \& Filacchione, G. 2014, Icarus, 237, 293

Ciarniello, M., Capaccioni, F., Filacchione, G., et al. 2015, A\&A, 583, A31

Ciarniello, M., De Sanctis, M. C., Ammannito, E., et al. 2017, A\&A, 598, A130

Clark, B. E., Veverka, J., Helfenstein, P., et al. 1999, Icarus, 140, 53

Combe, J.-P., McCord, T. B., Tosi, F., et al. 2016, Science, 353, aaf3010

De Sanctis, M. C., Coradini, A., Ammannito, E., et al. 2011, Space Sci. Rev., 163, 329

De Sanctis, M. C., Ammannito, E., Raponi, A., et al. 2015, Nature, 528, 241
De Sanctis, M. C., Raponi, A., Ammannito, E., et al. 2016, Nature, 536, 54 De Sanctis, M. C., Ammannito, E., McSween, H. Y., et al. 2017, Science, 355, 719

De Sanctis, M. C., Vinogradoff, V., Raponi, A., et al. 2018, MNRAS, 482, 2407

Déau, E., Dones, L., Charnoz, S., et al. 2013, Icarus, 226, 591

Devogèle, M., Tanga, P., Cellino, A., et al. 2018, Icarus, 304, 31

Domingue, D. L., Robinson, M., Carcich, B., et al. 2002, Icarus, 155, 205

Hapke, B. 1993, Theory of Reflectance and Emittance Spectroscopy, Topics in Remote Sensing (Cambridge, UK: Cambridge University Press), 3

Hapke, B. 2012, Theory of Reflectance and Emittance Spectroscopy (Cambridge, UK: Cambridge University Press)

Hapke, B., \& Sato, H. 2016, Icarus, 273, 75

Hapke, B., \& Van Horn, H. 1963, J. Geophys. Res., 68, 4545

Hasselmann, P. H., Barucci, M. A., Fornasier, S., et al. 2017, MNRAS, 469, S550 Helfenstein, P., \& Shepard, M. K. 2011, Icarus, 215, 83

Helfenstein, P., \& Veverka, J. 1989, in Asteroids II, eds. R. Binzel, T. Gehrels, \& M. Matthews (Tucson, AZ: University of Arizona Press), 557

Helfenstein, P., Veverka, J., \& Hillier, J. 1997, Icarus, 128, 2

Helfenstein, P., Currier, N., Clark, B., et al. 1998, Icarus, 135, 41

Jost, B., Pommerol, A., Poch, O., et al. 2017, Planet. Space Sci., 148, 1

Kaasalainen, S., Peltoniemi, J., Näränen, J., et al. 2005, Appl. Opt., 44, 1485

Kaplan, H. H., Milliken, R. E., \& Alexander, C. M. O. 2018, Geophys. Res. Lett., 45,5274

Kitazato, K., Clark, B. E., Abe, M., et al. 2008, Icarus, 194, 137

Li, J.-Y., Schröder, S. E., Mottola, S., et al. 2019, Icarus, 322, 144

Longobardo, A., Palomba, E., Galiano, A., et al. 2019, Icarus, 320, 97

Masoumzadeh, N., Boehnhardt, H., Li, J.-Y., \& Vincent, J.-B. 2015, Icarus, 257, 239

Mishchenko, M. I. 1992, Ap\&SS, 194, 327

Mishchenko, M. I., Dlugach, J. M., Liu, L., et al. 2009, ApJ, 705, L118

Muinonen, K. 1990, Light scattering by inhomogeneous media: backward enhancement and reversal of linear polarization, $\mathrm{PhD}$ thesis, University of Helsinki, Finland

Müller, T. G., \& Lagerros, J. S. V. 1998, A\&A, 338, 340

Nelson, R. M., Hapke, B. W., Smythe, W. D., \& Spilker, L. J. 2000, Icarus, 147, 545

Nelson, R. M., Smythe, W. D., Hapke, B. W., \& Hale, A. S. 2002, Planet. Space Sci., 50, 849

Palomba, E., Longobardo, A., De Sanctis, M. C., et al. 2019, Icarus, 320, 202

Pilorget, C., Fernando, J., Ehlmann, B. L., Schmidt, F., \& Hiroi, T. 2016, Icarus, 267, 296

Pitman, K. M., Buratti, B. J., \& Mosher, J. A. 2010, Icarus, 206, 537

Raponi, A. 2015, ArXiv e-prints [arXiv:1503.08172]

Raponi, A., De Sanctis, M. C., Frigeri, A., et al. 2018, Sci. Adv. 4, eaao3757

Raponi, A., De Sanctis, M. C., Carrozzo, F. G., et al. 2019a, Icarus, 320, 83

Raponi, A., Carrozzo, F. G., Zambon, F., et al. 2019b, Icarus, 318, 99

Reddy, V., Li, J.-Y., Gary, B. L., et al. 2015, Icarus, 260, 332

Rognini, E., Capria, M., Tosi, F., et al. 2019, J. Geophys. Res. Planets, 124

Rosenbush, V., Kiselev, N., \& Avramchuk, V. 2006, J. Quant. Spectr. Rad. Transf., 100, 325

Rousseau, B., Raponi, A., Ciarniello, M., et al. 2019, Rev. Sci. Instrum., 90, 121401

Salo, H., \& French, R. G. 2010, Icarus, 2010, 785

Schröder, S. E., Grynko, Y., Pommerol, A., et al. 2014, Icarus, 239, 201

Schröder, S. E., Mottola, S., Carsenty, U., et al. 2017, Icarus, 288, 201

Schröder, S. E., Li, J.-Y., Rayman, M. D., et al. 2018, A\&A, 620, A201

Shepard, M. K., \& Helfenstein, P. 2011, Icarus, 215, 526

Shevchenko, V. G., Belskaya, I. N., Chiorny, V. G., et al. 1997, Planet. Space Sci., 45,1615

Shevchenko, V. G., Belskaya, I. N., Muinonen, K., et al. 2016, Planet. Space Sci., 123, 101

Shkuratov, Y. 1989, Solar Syst. Res., 23, 111

Shkuratov, Y. G., \& Helfenstein, P. 2001, Icarus, 152, 96

Shkuratov, Y., Kreslavsky, M. A., Ovcharenko, A. A., et al. 1999, Icarus, 141, 132

Shkuratov, Y., Ovcharenko, A., Zubko, E., et al. 2004, J.Quant. Spectr. Rad. Transf., 88, 267

Shkuratov, Y., Kaydash, V., Korokhin, V., et al. 2011, Planet. Space Sci., 59, 1326

Sierks, H., Keller, H. U., Jaumann, R., et al. 2011, Space Sci. Rev., 163, 263

Spjuth, S., Jorda, L., Lamy, P. L., Keller, H. U., \& Li, J.-Y. 2012, Icarus, 221, 1101

Stankevich, D. G., Shkuratov, Y. G., \& Muinonen, K. 1999, J. Quant. Spectr. Rad. Transf., 63, 445

Tedesco, E. F., Williams, J. G., Matson, D. L., et al. 1989, AJ, 97, 580

Tholen, D. J. 1989, in Asteroids II, eds. R. P. Binzel, T. Gehrels, \& M. S. Matthews (Tucson, AZ: University of Arizona Press), 1139 


\section{Appendix A: Comparison with Monte Carlo simulations}

With the goal of further characterizing the OE surge on Ceres, we compare the derived phase curve with reflectance models from Monte Carlo ray-tracing simulations. To produce the reflectance models, we apply the routine described in Ciarniello et al. (2014). This allows us to simulate a particulate medium of spherical grains whose physical properties are defined in terms of the SPPF (modeled with a three-parameter Henyey-Greenstein function, Eq. (3)) and SSA. In addition, the overall porosity of the medium can be varied. For the purpose of this work, we assume a medium composed of particles all of the same size. Because numerical simulations are time-consuming, we are not able to directly simulate the reflectance output for all the VIR observations. A reasonable approach is therefore to select a limited number of observation geometries that properly sample the phase curve. An additional constraint is given by the fact that our Monte Carlo ray-tracing routine cannot handle large-scale roughness of the simulated material. This limits the selection to observations for which the effect of surface roughness and relative shadowing can be considered small. In this respect, observations at a low incidence angle assure a minor contribution of shadows. Moreover, at least according to Hapke's model, the effect of surface roughness on the reflectance increases for large phase and emission angles, thus preventing us from simulating these configurations. From this reasoning, we can define the following reference observation geometries, characterized by null incidence angle $i=0^{\circ}$ and $e=\alpha$ with $\alpha=0^{\circ}, 0.2^{\circ}, 0.5^{\circ}$, $1^{\circ}, 2^{\circ}, 3^{\circ}, 4^{\circ}, 5^{\circ}, 7^{\circ}, 10^{\circ}, 13^{\circ}, 16^{\circ}, 19^{\circ}, 25^{\circ}$, which satisfy the criteria discussed above and provide a good sampling of the phase curve in the $\mathrm{OE}$ region.

A comparison of the simulated reflectance curves with selected pixels at these exact observation geometries would be impractical, given the instrumental error in the observations, the effect of the intrinsic albedo variability on the surface, and the limited number of available data at a particular geometry. We therefore compare the simulated reflectance curve phase with the prediction at the same combination of $i, e, \alpha$ provided by Hapke's model using the parameters of S2, which represents the most accurate solution among those described in Sect. 3. Reflectance curves are derived for different simulated media corresponding to all the possible combinations of the values of SSA, $b_{1}, b_{2}, c$ and $P$ indicated below:

- SSA $\in[0.01,0.4]$ with 0.01 steps;

$-b_{1}=[0.3,0.5,0.6]$

$-b_{2}=[0,0.1,0.2,0.3,0.5]$

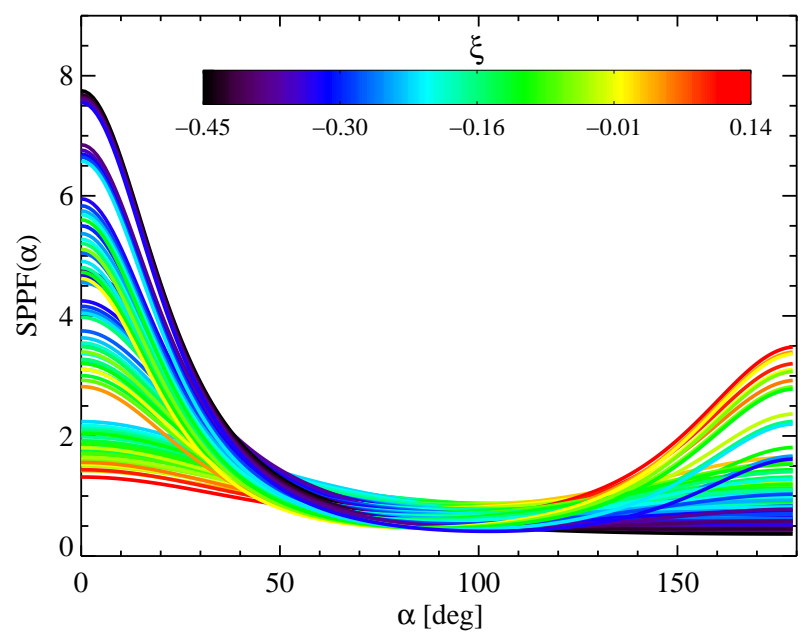

Fig. A.1. Single particle phase functions (SPPF) for all the investigated combinations of $b_{1}, b_{2}$, and $c$. The color bar represents the corresponding asymmetry parameter.

$-c=[-0.1,0 ., 0.1,0.3,0.5]$

$-P=[0.7,0.8,0.9,0.92,0.95]$

for a total of 15000 different models. The selected values of $b_{1}, b_{2}, c$ provide SPPFs with varying photometric behavior, from slightly forward-scattering to markedly back-scattering (Fig. A.1). Moreover, for a given porosity and SSA, the results of the simulations are interpolated in the $b_{1}, b_{2}, c$ parameter space to explore the effect of intermediate values which were not directly simulated. Each set of simulations for a given value of porosity $P$ is then compared to the reference Hapke model, and the best combination of parameters is derived using a least square method. Figure A.2 shows the best fits of Ceres' phase curve for the different investigated porosities. Interestingly, it can be noted that the best match is achieved for $P=0.9-0.92$. This independently confirms the same value derived in Sect. 3.3.4 by means of Hapke's model, which points to high porosity on Ceres' surface. Nonetheless, we stress here that such a result is based on the assumption that light scattering on Ceres can be described in the geometric optics regime, and that $\mathrm{CB}$, which is not modeled explicitly in our simulations, is not the dominant process in producing the OE. Apart from porosity, we are not able to provide robust constraints on the other parameters involved in the simulations, such as the SSA and the ones governing the SPPF's shape, given the limited phase angle range explored here. 

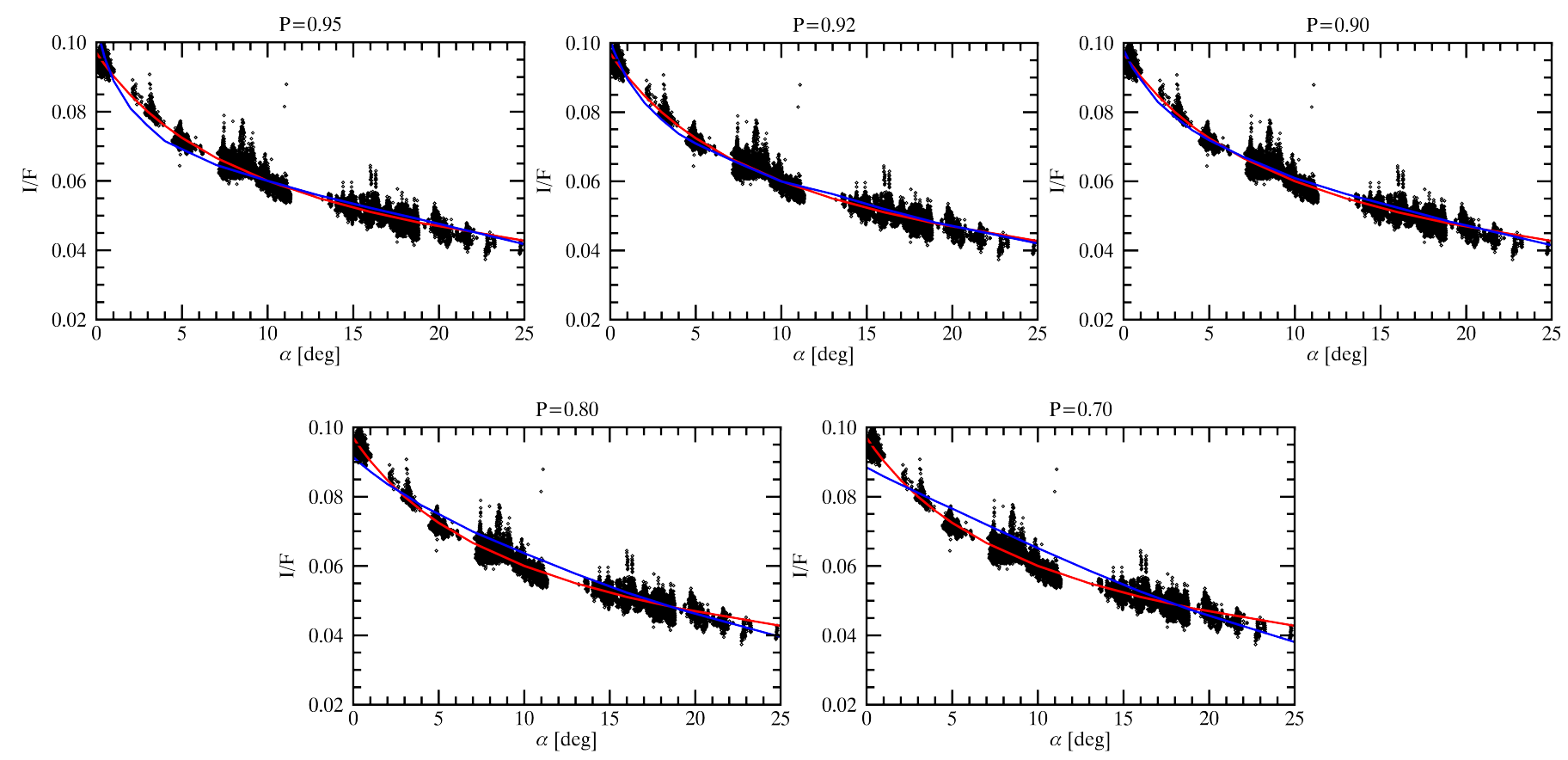

Fig. A.2. Best fit of Ceres' phase curve with Monte Carlo simulations for terrains with different porosities $P$. In each plot, diamonds represent VIR data, the blue curve is from Monte Carlo simulations, and the red one is the output from the Hapke' model with S2 parameters used as a reference for Ceres' photometric behavior. 